\begin{tabular}{|r|l|}
\hline \multicolumn{2}{|c|}{ Statistica Sinica Preprint No: SS-2020-0343 } \\
\hline Title & Large Sample Properties of Matching for Balance \\
\hline Manuscript ID & SS-2020-0343 \\
\hline URL & http://www.stat.sinica.edu.tw/statistica/ \\
\hline DOI & $10.5705 /$ ss.202020.0343 \\
\hline Complete List of Authors & Yixin Wang and \\
& José Zubizarreta \\
\hline Corresponding Author & José Zubizarreta \\
\hline E-mail & zubizarreta@hcp.med.harvard.edu \\
\hline Notice: Accepted version subject to English editing.
\end{tabular}




\title{
LARGE SAMPLE PROPERTIES OF MATCHING FOR BALANCE
}

\author{
Yixin Wang $^{1}$ and José R. Zubizarreta ${ }^{2}$ \\ ${ }^{1}$ University of Michigan and ${ }^{2}$ Harvard University
}

Abstract: Matching methods are widely used for causal inference in observational studies. Among them, nearest neighbor matching is arguably the most popular. However, nearest neighbor matching does not generally yield an average treatment effect estimator that is consistent at the $\sqrt{n}$ rate (Abadie and Imbens, 2006). Are matching methods not $\sqrt{n}$-consistent in general? In this paper, we study a recent class of matching methods that use integer programming to directly target aggregate covariate balance, in addition to finding close neighbor matches. We show that under suitable conditions these methods can yield simple estimators that are $\sqrt{n}$-consistent and asymptotically optimal. 
Key words and phrases: Causal Inference; Integer Programming;

Matching Methods; Observational Studies; Propensity Score.

\section{Introduction}

In observational studies, matching methods are widely used for causal inference. The great appeal of matching methods lies in the transparency of their covariate adjustments. These adjustments are an interpolation based on the available data rather than an extrapolation based on a potentially misspecified model (Rubin, 1973; Rosenbaum, 1989; Abadie \& Imbens, 2006). The structure of the data after matching is also simple (often, a self-weighted sample) so that statistical inferences and sensitivity analyses are straightforward (Rosenbaum, 2002, 2010, 2017). Matching methods are commonly used under the identification assumption of strong ignorability Rosenbaum \& Rubin, 1983) or selection on observables (Imbens \& Wooldridge, 2009), but they are also used under the different assumptions required by instrumental variables (e.g., Baiocchi et al. 2010) and discontinuity designs (e.g., Keele et al. 2015).

While there is an extensive literature on matching methods, large sam- 
ple characterizations of matching estimators have centered around nearest neighbor matching only (Abadie \& Imbens, 2006, 2011). In its simplest form, this algorithm matches each treated unit to the closest available control in terms of a covariate distance (e.g., the Mahalanobis distance; Rubin 1973). In an important paper, Abadie \& Imbens (2006) showed that the resulting difference-in-means estimator is not in general $\sqrt{n}$-consistent for the average treatment effect when matching with replacement. This estimator contains a bias that decreases at a rate inversely proportional to the number of covariates used for matching. As a result, its convergence can be very slow when matching on many covariates.

Different variants of nearest neighbor matching have been proposed to address this issue. In one variant, Abadie \& Imbens (2011) proposed a class of bias-corrected matching estimators where the missing potential outcomes are imputed with a regression model. This imputation corrects the bias of classical nearest neighbor matching. In another variant, Abadie \& Imbens (2016) formalized matching on the estimated propensity score. The estimated propensity score reduces the matching space into a single dimension. All of these variants achieve $\sqrt{n}$-consistency. However, in these cases the faster convergence rate depends on specifying correctly either the 
treatment or the outcome model, or restricting the outcome model to be Lipschitz continuous on the covariates.

Here, we study a recent class of optimization-based matching methods that directly target aggregate covariate balance and do not explicitly model the treatment or the outcome (Zubizarreta, 2012; Diamond \& Sekhon, 2013; Nikolaev et al., 2013; Zubizarreta et al., 2014). These methods formulate the matching exercise as an integer programming problem. For instance, cardinality matching (Zubizarreta et al. 2014) optimizes the number of matched treated and control units subject to constraints that approximately balance the empirical distributions of the covariates. We show that, under suitable conditions, the resulting difference-in-means treatment effect estimator is $\sqrt{n}$-consistent, asymptotically Normal, and semiparametrically efficient. These results show that matching for aggregate covariate balance can be asymptotically optimal when nearest neighbor matching with replacement is not. To our knowledge, this is the first work to show that a matching estimator can be semiparametrically efficient under suitable conditions.

To perform this asymptotic analysis of matching for balance, we establish a connection between matching and weighting, and view matching as a form of weighting for covariate balance that both weights treatment 
and control units and encodes an assignment between them. Examples of weighting methods for covariate balance include Hainmueller (2012), Imai \& Ratkovic (2014), Zubizarreta (2015), Chan et al. (2016), Fan et al. (2016), Zhao \& Percival (2017), Athey et al. (2018), Hirshberg \& Wager (2018), Zhao (2019), and Wang \& Zubizarreta (2020). This connection between matching and weighting enables us to analyze matching for balance using asymptotic techniques developed for weighting.

Despite its connection to weighting, matching for balance retains some essential features of nearest neighbor matching and other optimal covariate distance matching algorithms (e.g., Hansen 2004). In a similar way to distance matching algorithms, matching for balance can also focus on forming close unit matches in addition to achieving covariate balance in aggregate. In fact, matching for balance can be followed by re-matching for homogeneity in order to not only preserve aggregate covariate balance in the matched sample, but also minimize total covariate distances between its matched units (see Zubizarreta et al. 2014 for details). As discussed by Rosenbaum (2005) and Visconti \& Zubizarreta (2018), re-matching for homogeneity can improve the efficiency and sensitivity of certain matching estimators to unobserved covariates. The main message of this paper is 
that matching for balance (along with re-matching for homogeneity) can improve the large sample properties of the classical difference-in-means estimator in causal inference, achieving asymptotic optimality under suitable conditions.

This paper is organized as follows. In Section 2 we describe the identification assumptions, matching methods, and the matching estimator. In Section 3 we present and discuss our main results. In Section 4 we evaluate the empirical performance of the estimator. In Section 5 we conclude with some remarks. All proofs are shown in the Supplementary Materials.

\section{Matching for aggregate covariate balance}

In this section, we describe the causal estimation problem and introduce a class of matching methods that target aggregate covariate balance. We use the potential outcomes framework for causal inference Neyman, 1990; Rubin, 1974). With binary treatments, this framework posits that each unit $i=1, \ldots, N$ has a pair of potential outcomes $\left\{Y_{i}(0), Y_{i}(1)\right\}$, where $Y_{i}(1)$ is realized if unit $i$ is assigned to treatment $\left(Z_{i}=1\right)$ and $Y_{i}(0)$ is realized if the unit is assigned to control $\left(Z_{i}=0\right)$. Thus, for each unit $i$, we observe either $Y_{i}(0)$ or $Y_{i}(1)$, and the observed outcome writes $Y_{i}=Z_{i} Y_{i}(1)+\left(1-Z_{i}\right) Y_{i}(0)$. 
In our setting, the units $i=1, \ldots, N$ are a random sample from a population of interest and thus the potential outcomes are viewed as random variables.

Denote $X_{i}$ as the vector of observed covariates of unit $i$. These covariates can be continuous or discrete. Given these covariates, we assume strong ignorability of the treatment assignment (Rosenbaum \& Rubin, 1983): $Z_{i} \Perp\left\{Y_{i}(0), Y_{i}(1)\right\} \mid X_{i}$, and $0<\operatorname{Pr}\left(Z_{i}=1 \mid X_{i}\right)<1$. As implied by our notation, we also require the stable unit treatment value assumption (SUTVA; Rubin 1980).

The goal is to estimate the average treatment effect (ATE),$\mu=\mathbb{E}\left[Y_{i}(1)-\right.$ $\left.Y_{i}(0)\right]$. We choose this goal for notational convenience only. For example, our arguments for consistent and efficient estimation of the ATE can be directly extended to the average treatment effect on the treated (ATT), $\mu_{t}=\mathbb{E}\left[Y_{i}(1)-Y_{i}(0) \mid Z_{i}=1\right]$

We study matching methods that directly balance the empirical distributions of the observed covariates. Examples of these methods are $\mathrm{Zu}-$ bizarreta (2012), Diamond \& Sekhon (2013), and Nikolaev et al. (2013); other related examples include Fogarty et al. (2016), Pimentel et al. (2015) and Kallus (2020). At a high level, these methods aim to balance the covariates or certain transformations of them that span a function space (see 
Wang \& Zubizarreta 2020 for a discussion). We call these matching methods matching for balance. Extending the formulation in Zubizarreta et al. (2014), we study the following matching method

$\max . \quad M$

s.t. $\quad m_{i j} \in\{0,1\}, \quad i, j=1, \ldots, n$,

$\sum_{j=1}^{n}\left(1-Z_{j}\right) m_{i j}=M, \quad \forall i \in\left\{i: Z_{i}=1\right\}$,

$\sum_{i=1}^{n} Z_{i} m_{i j}=M, \quad \forall i \in\left\{j: Z_{j}=0\right\}$,

$$
\sum_{i=1}^{n} \sum_{j=1}^{n} Z_{i} Z_{j} m_{i j}=\sum_{i=1}^{n} \sum_{j=1}^{n}\left(1-Z_{i}\right)\left(1-Z_{j}\right) m_{i j}=0
$$

$\left|\sum_{i=1}^{n} \sum_{j=1}^{n} \frac{Z_{i}\left(1-Z_{j}\right) m_{i j}\left\{B_{k}\left(X_{i}\right)-B_{k}\left(X_{j}\right)\right\}}{\sum_{i=1}^{n} \sum_{j=1}^{n} Z_{i}\left(1-Z_{j}\right) m_{i j}}\right|<\delta_{k}, k \in[K]$

$$
\left|\sum_{i=1}^{n} \sum_{j=1}^{n} \frac{\left(1-Z_{i}\right) Z_{j} m_{i j}\left\{B_{k}\left(X_{i}\right)-B_{k}\left(X_{j}\right)\right\}}{\sum_{i=1}^{n} \sum_{j=1}^{n}\left(1-Z_{i}\right) Z_{j} m_{i j}}\right|<\delta_{k}, k \in[K]
$$

where $m_{i j}$ is a binary decision variable that indicates whether unit $i$ is matched to unit $j$ (Equation (2.2). Equations (2.3) and (2.4) require each treated unit be matched to $M$ control units, and each control unit be matched to $M$ treated units, respectively. Equation 2.5 enforces that each treated unit is not matched to another treated unit, nor that a control unit is matched to another control unit; in other words, only matches 
between different treatment groups are allowed. Finally, Equations 2.6 and (2.7) ensure that the covariate distributions of the matched treated and control units are balanced. In these constraints, the functions $B_{k}(\cdot)$ are suitable transformations of the covariates. Each of them maps the multivariate covariate vector $X_{i}$ into a suitable summary scalar. For example, they can be polynomials or wavelets. Thus, Equations $(2.6)$ and $(2.7)$ constrain the imbalances in these basis functions in the matched sample up to a level $\delta_{k}$. The constant $\delta_{k}$ is a tuning parameter chosen by the investigator. Zhao (2019) and Wang \& Zubizarreta (2020) describe algorithms to automatically select the tuning parameter $\delta_{k}$ in covariate balance optimization problems like (2.1)-(2.7).

As a whole, optimization problem (2.1)- 2.7$)$ finds the largest matched sample with replacement that is balanced according to the conditions specified in Equation (2.6) and Equation (2.7). An interesting feature of this approach is that it accomplishes the task of matching with replacement without predefining the $1: M$ matching ratio, but instead optimizing $M$ from the data at hand subject to the aggregate covariate balance constraints. We may posit additional constraints in order to match without replacement: $\sum_{i=1}^{n} Z_{i} m_{i j} \leq 1, \forall j \in\left\{j: Z_{j}=0\right\}$. In the asymptotic analyses 
below, we focus on matching with replacement, but these analyses can be extended to matching without replacement.

Of course, the above optimization problem of matching for balance may be infeasible. For example, this will be the case under practical violations of the positivity assumption; or more specifically, if there is limited overlap in covariate distributions as characterized by the functions $B_{k}(\cdot)$. In this case, one is left with the choice to either base the covariate adjustments on a model that goes beyond the support of the data or to discard some units and possibly change the target of inference (Crump et al., 2009). In this regard, the infeasibility certificate of the matching for balance problem provides valuable information to characterize the data at hand. In the Supplementary Materials, we provide sufficient conditions that guarantee the existence of a solution to the matching for balance optimization problem.

In order to estimate the ATE with matching for balance, we use a simple difference-in-means estimator:

$$
\begin{aligned}
\hat{\mu}:=\frac{1}{n}\left[\sum_{i=1}^{n} Z_{i}\left\{Y_{i}-\frac{\sum_{j=1}^{n}\left(1-Z_{j}\right) m_{i j} Y_{j}}{\sum_{j=1}^{n}\left(1-Z_{j}\right) m_{i j}}\right\}\right. \\
\left.+\sum_{i=1}^{n}\left(1-Z_{i}\right)\left(\frac{\sum_{j=1}^{n} Z_{j} m_{i j} Y_{j}}{\sum_{j=1}^{n} Z_{j} m_{i j}}-Y_{i}\right)\right] .
\end{aligned}
$$


This estimator computes the average difference between each unit and its matches. For example, the first term of Equation (2.8) is the difference between the outcome of each treated unit $Y_{i}$ and the mean outcome of the units it is matched to, $\left\{Y_{j}: m_{i j}=1, Z_{j}=0\right\}$. Analogously, the second term is the difference between the outcome of each control unit $Y_{i}$ and the mean outcome of its matches, $\left\{Y_{j}: m_{i j}=1, Z_{j}=1\right\}$.

Using Equation (2.3) and Equation (2.4), we can rewrite this differencein-means estimator as

$$
\begin{aligned}
\hat{\mu}=\frac{1}{n}\left[\left\{\sum_{i=1}^{n} Z_{i} Y_{i}\right.\right. & \left.+\sum_{j=1}^{n} \frac{\sum_{i=1}^{n}\left(1-Z_{i}\right) m_{i j}}{M} Z_{j} Y_{j}\right\} \\
& \left.-\left\{\sum_{i=1}^{n}\left(1-Z_{i}\right) Y_{i}+\sum_{j=1}^{n} \frac{\sum_{i=1}^{n} Z_{i} m_{i j}}{M}\left(1-Z_{j}\right) Y_{j}\right\}\right] .
\end{aligned}
$$

This form implies that each unit $j$ receives weight $\left\{1+\sum_{i=1}^{n}\left(1-Z_{i}\right) m_{i j} / M\right\}$ if it is treated and weight $\left\{1+\sum_{i=1}^{n} Z_{i} m_{i j} / M\right\}$ if it is a control. Using Equation (2.3) and Equation (2.4), we can also rewrite the covariate balance constraints in Equation (2.6) as

$$
\begin{array}{r}
\frac{1}{\sum_{i=1}^{n} Z_{i}}\left|\sum_{i=1}^{n} Z_{i} B_{k}\left(X_{i}\right)-\sum_{j=1}^{n} \frac{\sum_{i=1}^{n} Z_{i} m_{i j}}{M}\left(1-Z_{j}\right) B_{k}\left(X_{j}\right)\right|<\delta_{k}, \\
\frac{1}{\sum_{i=1}^{n}\left(1-Z_{i}\right)}\left|\sum_{i=1}^{n}\left(1-Z_{i}\right) B_{k}\left(X_{i}\right)-\sum_{j=1}^{n} \frac{\sum_{i=1}^{n}\left(1-Z_{i}\right) m_{i j}}{M} Z_{j} B_{k}\left(X_{j}\right)\right|<\delta_{k} .
\end{array}
$$


We observe that the weights of the units in both the constraints and the ATE estimator are functions of the frequencies they are matched, namely

$$
\begin{aligned}
& w_{T}\left(X_{j}\right)=\frac{\sum_{i=1}^{n}\left(1-Z_{i}\right) m_{i j}}{M} \quad \text { if } X_{j} \text { is treated, } \\
& w_{C}\left(X_{j}\right)=\frac{\sum_{i=1}^{n} Z_{i} m_{i j}}{M} \quad \text { if } X_{j} \text { is control. }
\end{aligned}
$$

We note that the numerator and denominator of the weights $w_{T}\left(X_{j}\right)$ and $w_{C}\left(X_{j}\right)$ must be integers due to Equations $(2.2)$ and $(2.3)$. This restricts the values that the weights can take. Besides this constraint, the integer program for matching resembles the convex optimization problem in covariate balancing weights (Zhao, 2019; Wang \& Zubizarreta, 2020).

This connection between matching and weighting allows us to establish the asymptotic optimality of matching for balance. In the following section, we show that under suitable conditions, the above difference-in-means ATE estimator is $\sqrt{n}$-consistent, asymptotically Normal, and semiparametrically efficient. As mentioned, we focus on the ATE for simplicity of exposition. These consistency and asymptotic normality results readily extend to the ATT because the integer programming problem for the ATT is analogous to that of the ATE. The difference is that we match control units to each treated unit, but not treated units to controls. We note that calculating the 
asymptotic variance of the ATT estimator is more nuanced. In particular, the semiparametric efficiency bound depends on whether we know the true model for the propensity score.

\section{Asymptotic properties of matching for balance}

In this section, we show that under standard assumptions, matching for balance is asymptotically optimal: the resulting ATE estimator is $\sqrt{n}$ consistent, asymptotically Normal, and semiparametrically efficient.

We start by describing the assumptions required. We posit three sets of conditions on the basis functions $B(x)=\left(B_{1}(x), \ldots, B_{K}(x)\right)^{\top}$, the propensity score function $\pi(x)=\operatorname{Pr}\left(Z_{i}=1 \mid X_{i}=x\right)$, and the mean potential outcome functions $\mathbb{E}\left[Y_{i}(z) \mid X_{i}=x\right]$ for $z \in\{0,1\}$.

Assumption 1. Assume the following conditions on the basis functions $B(x)=\left(B_{1}(x), \ldots, B_{K}(x)\right)^{\top}$ hold. There exist constants $C_{0}, C_{1}, C_{2}>0$ such that:

1. $\sup _{x \in \mathcal{X}}\|B(x)\|_{2} \leq C_{0} K^{1 / 2}$, where $\mathcal{X}$ is the domain of the covariates $X$, which is compact.

2. $\left\|\mathbb{E}\left[B\left(X_{i}\right)^{\top} B\left(X_{i}\right)\right]\right\|_{2} \leq C_{1}$. 
3. $\lambda_{\min }\left\{\mathbb{E}\left[B\left(X_{i}\right) B\left(X_{i}\right)^{\top}\right]\right\}>C_{2}$, where $\lambda_{\min }\left\{\mathbb{E}\left[B\left(X_{i}\right) B\left(X_{i}\right)^{\top}\right]\right\}$ denotes the smallest eigenvalue of the matrix $\mathbb{E}\left[B\left(X_{i}\right) B\left(X_{i}\right)^{\top}\right]$.

Assumptions 1, 1-1,3 are standard regularity conditions on the basis functions. They restrict their magnitude by the norm of the length- $K$ basis function vector. These conditions are common in nonparametric sieve estimation (see Assumption 4.1.6 of Fan et al. 2016 and Assumption 2(ii) of Newey 1997). They are satisfied by many classes of basis functions including regression spline, trigonometric polynomial, and wavelet bases (Newey, 1997; Horowitz et al., 2004; Chen, 2007; Belloni et al., 2015; Fan et al., 2016).

Assumption 2. Assume the following conditions on the propensity score function $\pi(x)=\operatorname{Pr}\left(Z_{i}=1 \mid X_{i}=x\right)$ hold.

1. There exists a constant $C_{3}>0$ such that $C_{3}<\pi(x)<1-C_{3}$.

2. There exist vectors $\left(\lambda_{1 T}^{*}\right)_{K \times 1},\left(\lambda_{1 C}^{*}\right)_{K \times 1}$ such that the true propensity score function $\pi(\cdot)$ satisfies $\sup _{x \in \mathcal{X}}\left|1 / \pi(x)-B(x)^{\top} \lambda_{1 T}^{*}\right|=O\left(K^{-r_{\pi}}\right)$ and $\sup _{x \in \mathcal{X}}\left|1 /\{1-\pi(x)\}-B(x)^{\top} \lambda_{1 C}^{*}\right|=O\left(K^{-r_{\pi}}\right)$, where $r_{\pi}>1$.

3. There exists a set $\mathcal{M}$ such that the propensity score function satisfies $1 / \pi(x) \in \mathcal{M}$ and $1 /\{1-\pi(x)\} \in \mathcal{M}$. Moreover, the set $\mathcal{M}$ is a set 
of smooth functions such that $\log n_{\square}\left\{\varepsilon, \mathcal{M}, L_{2}(\operatorname{Pr})\right\} \leq C_{4}(1 / \varepsilon)^{1 / k_{1}}$, where $n_{\square}\left\{\varepsilon, \mathcal{M}, L_{2}(\operatorname{Pr})\right\}$ denotes the covering number of $\mathcal{M}$ by $\varepsilon$ brackets, $L_{2}(\operatorname{Pr})$ is the norm defined as $\left\|m_{1}(\cdot)-m_{2}(\cdot)\right\|_{L_{2}(\operatorname{Pr})}=\mathbb{E}\left[m_{1}\left(X_{i}\right)-\right.$ $\left.m_{2}\left(X_{i}\right)\right]^{2}, C_{4}$ is a positive constant, and $k_{1}>1 / 2$.

Assumption 2.1 requires overlap between the treatment and control populations. This is part of the identification assumption described in Section 2. Assumption 2.2 is a smoothness condition on the inverse propensity score function. It requires the inverse propensity score be uniformly approximable by the basis functions $B(x)=\left(B_{1}(x), \ldots, B_{K}(x)\right)^{\top}$. For example, when we choose the basis functions to be multidimensional splines or power series, this assumption is satisfied for $r_{\pi}=s / d$, where $s$ is the number of continuous derivatives of $1 / \pi(x)$ and $d$ is the dimension of $x$, for $x$ with a compact domain $\mathcal{X}$ (Newey, 1997). Assumption 2.3 constrains the complexity of the function class to which the inverse propensity score function belongs. This assumption is satisfied, for example, by the Hölder class with smoothness parameter $s$ defined on a bounded convex subset of $\mathbb{R}^{d}$ with $s / d>1$ (Van Der Vaart \& Wellner, 1996; Fan et al., 2016). This is a key assumption that enables the use of empirical process techniques in establishing consistency and asymptotic normality. 
Assumption 3. Assume the following conditions on the mean potential outcome functions $Y_{z}(x) \triangleq \mathbb{E}\left[Y_{i}(z) \mid X_{i}=x\right]$ for $z \in\{0,1\}$ hold.

1. $\mathbb{E}\left|Y_{i}-Y_{0}\left(X_{i}\right)\right|<\infty$ and $\mathbb{E}\left|Y_{i}-Y_{1}\left(X_{i}\right)\right|<\infty$.

2. $|\mu|<\infty$, where $\mu=\mathbb{E}\left[Y_{i}(1)-Y_{i}(0)\right]$ is the true average treatment effect.

3. There exist $r_{y}>1 / 2,\left(\lambda_{2 C}^{*}\right)_{K \times 1}$, and $\left(\lambda_{2 T}^{*}\right)_{K \times 1}$ such that $\sup _{x \in \mathcal{X}} \mid Y_{0}(x)-$ $B(x)^{\top} \lambda_{2 C}^{*} \mid=O\left(K^{-r_{y}}\right)$ and $\sup _{x \in \mathcal{X}}\left|Y_{1}(x)-B(x)^{\top} \lambda_{2 T}^{*}\right|=O\left(K^{-r_{y}}\right)$.

4. The potential outcome functions satisfy $Y_{0}(\cdot) \in \mathcal{H}$ and $Y_{1}(\cdot) \in \mathcal{H}$, where $\mathcal{H}$ is a set of smooth functions satisfying $\log n_{[}\left\{\varepsilon, \mathcal{H}, L_{2}(\operatorname{Pr})\right\} \leq$ $C_{5}(1 / \varepsilon)^{1 / k_{2}}, C_{5}$ is a positive constant, and $k_{2}>1 / 2$. As in Assumption $23, n_{[]}\left\{\varepsilon, \mathcal{H}, L_{2}(\operatorname{Pr})\right\}$ denotes the covering number of $\mathcal{H}$ by $\varepsilon$-brackets, and $L_{2}(\operatorname{Pr})$ is the norm $\left\|m_{1}(\cdot)-m_{2}(\cdot)\right\|_{L_{2}(\operatorname{Pr})}=\mathbb{E}\left[m_{1}\left(X_{i}\right)-m_{2}\left(X_{i}\right)\right]^{2}$.

Assumptions 3,1 and 3,2 are regularity conditions on the mean potential outcomes. Assumptions 3.3 and 3.4 are analogous conditions to Assumptions 2.2 and 2.3 ; they constrain the smoothness of the mean potential outcome functions and the complexity of the function class they belong to. Under strong ignorability, one may get a rough sense of the function approximation quality (Assumption 2.3) by evaluating the prediction error 
of a fitted outcome model on a holdout sample. This model explains the observed outcomes in terms of the $K$ basis functions of the observed covariates plus the treatment assignment indicator. The approximation is likely to be good if the prediction error is small on the holdout data. That said, such an empirical evaluation will only give us a rough sense of the quality of the approximation, for we only have finite samples. We note that while no specific modeling assumptions are required for the inverse propensity score function and the potential outcome functions, Assumptions 2,2 and 2,3 do require both have the same form of smoothness, namely that they can all be well approximated by the same set of basis functions.

Assumption 4. Assume the following conditions on the matching for balance problem.

1. $K=o\left(n^{1 / 2}\right)$.

2. $\|\delta\|_{2}=O_{p}\left[K^{1 / 2}\left\{(\log K) / n+K^{-r_{\pi}}\right\}\right]$, where $\delta=\left(\delta_{1}, \ldots, \delta_{K}\right)$.

3. $n^{\frac{1}{2\left(r_{\pi}+r_{y}-0.5\right)}}=o(K)$, where $r_{\pi}, r_{y}$ are the smoothness parameters defined in assumptions Assumption 2,2 and Assumption 3.3.

Assumption 4.1 quantifies the rate at which the number of basis functions we balance can grow with the number of units. Assumption 42 limits 
the extent to which there can be imbalances in the basis functions. Despite these imbalances, we will show that the optimal large sample properties of the matching estimator is maintained. Assumption 4.3 characterizes the growth rates of $K$ and $n$ with respect to the uniform approximation rates $r_{\pi}$ and $r_{y}$.

Now we state the main result of this paper.

Theorem 3.1. Under Assumptions 1 to 4, the ATE estimator

$$
\begin{aligned}
\hat{\mu}:=\frac{1}{n}\left[\sum_{i=1}^{n} Z_{i}\left\{Y_{i}-\frac{\sum_{j=1}^{m}\left(1-Z_{j}\right) m_{i j} Y_{j}}{\sum_{j=1}^{m}\left(1-Z_{j}\right) m_{i j}}\right\}\right. \\
\left.+\sum_{i=1}^{n}\left(1-Z_{i}\right)\left\{\frac{\sum_{j=1}^{m} Z_{j} m_{i j} Y_{j}}{\sum_{j=1}^{m} Z_{j} m_{i j}}-Y_{i}\right\}\right]
\end{aligned}
$$

is $\sqrt{n}$-consistent, asymptotically Normal, and semiparametrically efficient:

$$
\sqrt{n}(\hat{\mu}-\mu) \stackrel{d}{\rightarrow} \mathcal{N}\left(0, V_{\text {opt }}\right)
$$

where $V_{\text {opt }}$ equals the semiparametric efficiency bound

$$
V_{\text {opt }}=\mathbb{E}\left[\frac{\operatorname{Var}\left[Y_{i}(1) \mid X_{i}\right]}{\pi\left(X_{i}\right)}+\frac{\operatorname{Var}\left[Y_{i}(0) \mid X_{i}\right]}{1-\pi\left(X_{i}\right)}+\left\{\mathbb{E}\left[Y_{i}(1)-Y_{i}(0) \mid X_{i}\right]-\mu\right\}^{2}\right]
$$

and $\pi\left(X_{i}\right)$ is the propensity score of unit $i$. If in addition $r_{y}>1$ holds, then 
the estimator

$$
\begin{aligned}
\hat{V}_{K}= & \sum_{i=1}^{n}\left[Z_{i} w\left(X_{i}\right) Y_{i}-\frac{\sum_{i=1}^{n} Z_{i} w\left(X_{i}\right) Y_{i}}{\sum_{i=1}^{n} Z_{i}}\right. \\
& -\left(1-Z_{i}\right) w\left(X_{i}\right) Y_{i}+\frac{\sum_{i=1}^{n}\left(1-Z_{i}\right) w\left(X_{i}\right) Y_{i}}{\sum_{i=1}^{n}\left(1-Z_{i}\right)} \\
& -\hat{Y}_{T}\left(X_{i}\right) \times\left\{Z_{i} w\left(X_{i}\right)-\frac{1}{\sum_{i=1}^{n} Z_{i}}\right\} \\
& \left.+\hat{Y}_{C}\left(X_{i}\right) \times\left\{\left(1-Z_{i}\right) w\left(X_{i}\right)-\frac{1}{\sum_{i=1}^{n}\left(1-Z_{i}\right)}\right\}\right]^{2} .
\end{aligned}
$$

is a consistent estimator of the asymptotic variance $V_{\text {opt }}$, where

$$
\begin{gathered}
\hat{Y}_{T}\left(X_{i}\right)=B\left(X_{i}\right)^{\top}\left\{\frac{\sum_{i=1}^{n} Z_{i} w\left(X_{i}\right) B\left(X_{i}\right)^{\top} B\left(X_{i}\right)}{\sum_{i=1}^{n} Z_{i}}\right\}^{-1} \\
\cdot\left\{\frac{\sum_{i=1}^{n} Z_{i} w\left(X_{i}\right) B\left(X_{i}\right) Y_{i}}{\sum_{i=1}^{n} Z_{i}}\right\} \\
\hat{Y}_{C}\left(X_{i}\right)=B\left(X_{i}\right)^{\top}\left\{\frac{\sum_{i=1}^{n}\left(1-Z_{i}\right) w\left(X_{i}\right) B\left(X_{i}\right)^{\top} B\left(X_{i}\right)}{\sum_{i=1}^{n}\left(1-Z_{i}\right)}\right\}^{-1} \\
\cdot\left\{\frac{\sum_{i=1}^{n}\left(1-Z_{i}\right) w\left(X_{i}\right) B\left(X_{i}\right) Y_{i}}{\sum_{i=1}^{n}\left(1-Z_{i}\right)}\right\} .
\end{gathered}
$$

Proof sketch. The proof uses empirical process techniques to analyze ATE estimators as in Fan et al. (2016) (see also Wang \& Zubizarreta 
2020). The key challenge in this proof lies in the need to characterize the entire class of matching solutions in matching for balance. More specifically, the optimization objective of matching for balance does not involve the matching solution $m_{i j}$ directly, so it does not correspond to a unique matching solution. We hence need to study the ATE estimates resulting from all possible matching solutions. In contrast, the balancing weights (Wang \& Zubizarreta, 2020) and the covariate balancing propensity score (Fan et al., 2016) both work with optimization objectives that involve all the weights; these problems also assume a unique weighting solution with infinite samples.

The proof starts by showing that the implied weights of matching for balance (Equation (2.10) approximate the true inverse propensity score function $\pi(x)^{-1}$. Moreover, this approximation is consistent due to the balancing constraints (Equations 2 2.6 $-(2.7)$ ). The rest of the proof involves a decomposition of $\hat{\mu}-\mu$ into seven components, where six of them converge to zero in probability, and the other one is asymptotically Normal and semiparametrically efficient. Each of the first six components can be controlled by the bracketing number of the function classes of the inverse propensity score and the outcome functions. Assumption 2.3 and Assumption 3 , 4 pro- 
vide this control. The full proof is in Appendix A of the Supplementary Materials.

An intuitive explanation of Theorem 3.1 relies on two observations. The first observation is that the ATE is an estimand derived from the entire population rather than from individual units. The asymptotic optimality of our ATE estimator depends primarily on whether the covariate distribution of the treated units is close in aggregate to that of the control units. For this type of estimator, how the individual units are matched to each other plays a secondary role. More specifically, the ATE estimator only depends on the number of times each treated (or control) unit is matched. For this reason, aggregate covariate balance is sufficient for the asymptotic optimality of matching estimators for the ATE. Matching for balance precisely targets this aggregate covariate balance. Equations 2.6 and 2.7 ensure that the covariate distributions after matching are balanced in aggregate for the treated and control units.

The second observation is the connection between matching for balance and covariate balancing weights (Hainmueller, 2012; Imai \& Ratkovic, 2014; Zubizarreta, 2015; Chan et al., 2016; Fan et al., 2016; Zhao \& Percival, 2017; Zhao, 2019, Wang \& Zubizarreta, 2020). Both methods formulate 
the estimation problem as a mathematical program under similar covariate balancing constraints as in Equations (2.6) and (2.7). Covariate balancing weights have been shown to be asymptotically optimal. Thus, if matching for balance admits a solution, its implied weights as in Equation 2.10 are as good as the covariate balancing weights. For this reason, under the conditions required by Assumptions 1 to 4, matching for balance can also be asymptotically optimal for the difference-in-means estimators for the ATE. When these assumptions do not hold, the nearest neighbor matching estimator for the ATE (which is a competing semiparametric estimator) does not similarly achieve the semiparametric efficiency bound.

We conclude this section with a discussion of Theorem 3.1 and its assumptions. Unlike other matching methods that assume a correct propensity or outcome model, Theorem 3.1 studies matching for balance that posits explicit conditions on covariate balance. Such conditions are practically appealing because covariate balance is what is typically checked in practice. Other regularity conditions and smoothness conditions are standard in nonparametric sieve estimation.

Under these conditions, Theorem 3.1 establishes the asymptotic optimality of the simple difference-in-means estimator for the ATE after match- 
ing for aggregate covariate balance. In practice, these conditions indicate (i) using basis functions such as power series or wavelets (Assumption 1), (ii) considering settings with smooth inverse propensity score and potential outcome functions, with more continuous derivatives than the number of covariates (Assumptions 2 and 3), and (iii) restricting the number of balancing basis functions $K$ and the approximate balance tolerance $\delta$ to scale appropriately with the number of samples (roughly, $K=O\left(n^{1 / 2-\epsilon}\right)$ and $\delta=O\left[K^{1 / 2}\left\{(\log K) / n+K^{-r_{\pi}}\right\}\right]$, where $\epsilon>0$ is a small number; Assumption 4. While Assumption 4 of Theorem 3.1 states that one may balance up to $K=O\left(n^{1 / 2-\epsilon}\right)$ basis functions as the sample size goes to infinity, in practice one should proceed with caution in any given finite sample. The matching for balance optimization problem may not admit a solution. Even if a solution exists, the finite sample performance of the resulting estimator may not be ideal. As Robins \& Ritov (1997) suggested, nonparametric estimators may suffer from the curse of dimensionality. There may not exist a nonparametric estimator with good finite sample performance without the knowledge of the true propensity score.

We note that Abadie \& Imbens (2011) also devise a matching estimator that is consistent at the $\sqrt{n}$-rate, but matching for balance achieves 
the $\sqrt{n}$-rate in a different way. Abadie \& Imbens (2011) correct the bias in nearest neighbor matching by positing a consistent regression model for the mean potential outcome function. In contrast, matching for balance avoids this bias by directly balancing the observed covariates in aggregate. Balancing covariates in aggregate has been shown to be equivalent to nonparametric estimation of the inverse propensity score and mean potential outcome functions (Fan et al., 2016; Zhao \& Percival, 2017; Hirshberg \& Wager, 2018; Zhao, 2019; Wang \& Zubizarreta, 2020). This nonparametric approach relieves us from positing a model for the mean potential outcome function that needs to be correctly specified. While Theorem 3.1 requires certain conditions on both the propensity score and the potential outcomes functions, it shows that the matching for balance estimator can achieve semiparametric efficiency, beyond $\sqrt{n}$-consistency.

Finally, Abadie \& Imbens (2012) provide a martingale representation of a widespread nearest neighbor matching estimator and derive its asymptotic distribution. They decompose the estimator into a martingale term and a conditional bias term. Both their and our analysis require the conditional bias term to vanish in order to achieve asymptotic consistency. Specifically, Abadie \& Imbens (2012) posit regularity conditions under which the condi- 
tional bias term converges in probability to zero. Theorem 3.1 utilizes the covariate balance conditions in Equations (2.6) and (2.7) to ensure that the conditional bias term vanishes.

\section{Simulation study}

Here, we illustrate the empirical performance of matching for balance. Our simulation study is based on a real dataset about the importance of market access for economic development (Redding \& Sturm, 2008). The covariates in this dataset are non-Gaussian and cannot be characterized by their first two moments. We focus on a setting where both the propensity score and the outcome are nonlinear functions of the covariates, and study the mean squared error (MSE) and coverage probabilities of matching for balance.

To generate the data, we take the actual covariate values from Redding \& Sturm (2008) and simulate the treatment and outcome values as follows. To simulate the treatment assignment indicator $Z_{i}$, we first fit a logistic regression model to the original indicator in the dataset. Specifically, we fit the model $\operatorname{Pr}\left(Z_{i}=1 \mid X_{i}\right)=\operatorname{sigmoid}\left\{\left(\alpha+\sum_{p=1}^{P} \beta_{j} X_{i p}+\right.\right.$ $\left.\left.\sum_{p, p^{\prime}=1}^{P} \beta_{p p^{\prime}} X_{i p} X_{i p^{\prime}}\right)\right\}$, where $X_{i p}$ denotes the $p$ th observed covariate of unit

$i$. We zero out the estimated coefficients with $p$-values smaller than 0.25 
and retain the rest of the coefficients. We finally generate the treatment assignment indicator $Z_{i}$ for the simulated dataset via a thresholding model $Z_{i}=\mathbf{1}\left\{Z_{i}^{*}>0\right\}$, where $Z_{i}^{*}=\left(\alpha+\sum_{p=1}^{P} \beta_{j} X_{i p}+\sum_{p, p^{\prime}=1}^{P} \beta_{p p^{\prime}} X_{i p} X_{i p^{\prime}}\right) / c+$ Unif $(-0.5,0.5)$, setting $c=50$ to induce limited overlap.

Next, we simulate the potential outcomes $\left\{Y_{i}(0), Y_{i}(1)\right\}$. Again, we begin by fitting a linear regression model with all possible second-order interaction terms to the original treated and control outcomes in the sample. The model is $Y_{i}=\alpha^{\prime}+\sum_{p=1}^{P} \beta_{j}^{\prime} X_{i p}+\sum_{p, p^{\prime}=1}^{P} \beta_{p p^{\prime}}^{\prime} X_{i p} X_{i p^{\prime}}+\beta_{t}^{\prime} T_{i}+\epsilon_{i}$, $\epsilon_{i} \sim N(0,1)$. As with the treatment assignment, we zero out all the estimated coefficients with $p$-values smaller than 0.25 , and predict the potential treated and control outcomes on the entire sample using the fitted model. We then generate the observed outcomes by $Y_{i}=Z_{i} Y_{i}(1)+\left(1-Z_{i}\right) Y_{i}(0)$. This data generating process yields a simulated dataset with the same size (122 units) as the original dataset in Redding \& Sturm (2008) and outcomes determined by three covariates and four associated relevant secondorder terms. Thus, if we balance up to the $k$ th univariate moment of the covariates, we need to balance $3 \cdot k$ basis functions.

With these simulated datasets, we evaluate the MSE of matching for balance in estimating the ATE. We vary the number of basis functions that 
we balance by setting the bases to be the moments of the covariates and increasing their order. We balance the first, second, and third moments of the covariates. We set the level of imbalance to be 0.1 standard deviations of the corresponding moment.

Figure 1 shows that as we increase the number of balancing basis functions, the MSE of matching for balance decreases. Also, because the simulated dataset has limited overlap, matching for balance achieves lower MSE than the standard augmented inverse propensity weighted estimator (AIPW; Robins et al. 1994) as the approximate balance constraints trade variance for bias. Nonetheless, when covariate distributions have limited overlap, this improvement in MSE comes at a cost. As we show below, the resulting confidence intervals may exhibit lower than nominal coverage due to approximate covariate balance. This suggests exploring separate imbalance tolerances for estimation and for inference. Finally, because of the nonlinearity of the inverse propensity score, including third order basis functions improves MSE despite the data generating process only involves second order terms.

Figure 1 also corroborates the discussion in Section 3 that matching for balance may not admit a solution if we aim to balance too many basis 
functions. For example, in Figure 1, matching for balance admits a solution if we balance the first three moments of the covariates, but not if we also try to balance the fourth moment for the given tolerance level of covariate imbalance. We note that in such cases, $\sqrt{n}$-consistency may not hold because the asymptotic results derived in Section 3 are only applicable when $K=o\left(n^{1 / 2}\right)$. These asymptotic results do not apply if matching for balance does not admit a solution when $K$ increases in this order and not enough matches can be found.

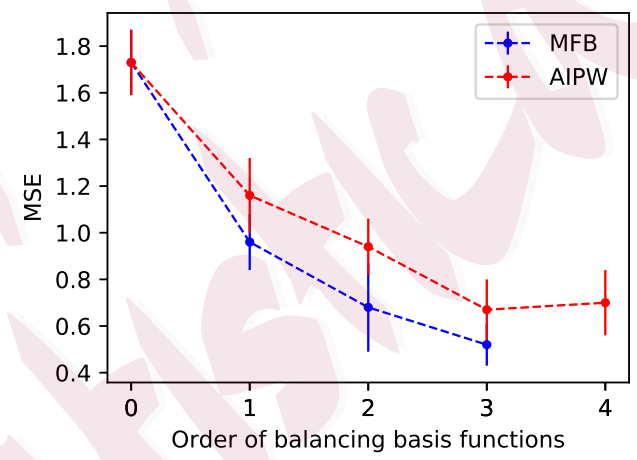

Figure 1: The matching for balance (MFB) estimator achieves lower MSE than the augmented inverse probability weighting (AIPW) estimator when the data has limited overlap. Increasing the number of balancing basis functions improves the MSE of MFB until its optimization problem becomes infeasible. The bars indicate \pm 1 standard deviation across 100 simulations.

Figure 2a shows that as we increase the number of balancing basis functions, the average number of matches $M$ decreases. The reason is that balancing more basis functions implies solving a more constrained opti- 
mization problem; hence, the average $M$ decreases. A similar phenomenon appears in Figure $2 \mathrm{~b}$ where the average standardized covariate balance

$$
\frac{1}{K} \sum_{k=1}^{K}\left[\left|\sum_{i=1}^{n} \sum_{p=1}^{n} \frac{Z_{i}\left(1-Z_{j}\right) m_{i j}\left\{B_{k}\left(X_{i}\right)-B_{k}\left(X_{j}\right)\right\}}{\sum_{i=1}^{n} \sum_{p=1}^{n} Z_{i}\left(1-Z_{j}\right) m_{i j}}\right| / \operatorname{sd}\left\{B_{k}\left(X_{i}\right)\right\}\right]
$$

increases with the number of balancing basis functions. (In Figure $2 \mathrm{~b}$, the order of balancing basis functions equal to zero represents covariate balance before matching.) While both metrics (the average number of matches and the average absolute standardized mean difference in covariates) decrease as we increase the number of basis functions that we balance, the MSE still improves because the treatment and control groups after matching are more similar in ways that are relevant to the propensity score and outcome models. This illustrates the importance of balancing more basis functions than just means when the propensity score and outcome models are nonlinear on the covariates. While balancing a high number of basis functions can be difficult with most matching methods (as they do not directly target covariate balance), with matching for balance, covariate balance on the basis functions is obtained by construction. Subject to these covariate balance requirements, the matching ratio $M$ in matching for balance is optimized, resulting in the largest possible $1: M / M: 1$ matching ratio for the data at 
hand.

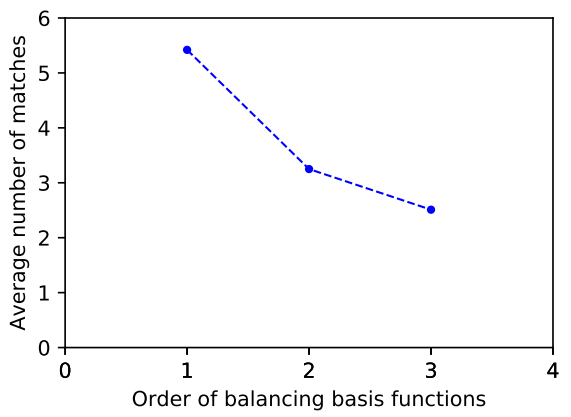

(a) Average number of matches.

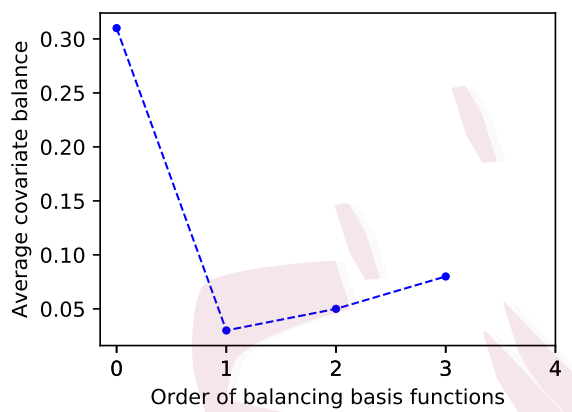

(b) Average covariate balance.

Figure 2: Average number of matches and average standardized covariate balance of matching for balance. While both measures decrease as we balance more basis functions, the MSE still improves because the covariate distributions of the treatment and control groups are more similar in ways that are relevant to the propensity score and outcome models.

Finally, we evaluate the coverage probabilities of matching for balance and AIPW. We focus on balancing the first two moments of the covariates and vary the imbalance tolerance $\delta$ such that $\delta_{k}=\delta \cdot \operatorname{sd}\left\{B_{k}\left(X_{i}\right)\right\}$. In Figure 3, we show the coverage probabilities of the $95 \%$ confidence intervals constructed based on Theorem 3.1. The figure shows that when the imbalance tolerance is small $(\delta \leq 0.01)$, the confidence intervals have close to nominal coverage. As the imbalance tolerance increases, the average number of matches increases but the coverage probability degrades as the matched sample exhibits worse balance. In contrast, the AIPW estimator achieves close to nominal coverage. In such cases, matching for balance 
trades the imbalance tolerance $\delta$ for the matching ratio $M$ in order to exchange bias for variance and achieve lower MSE, but it can compromise coverage probabilities.

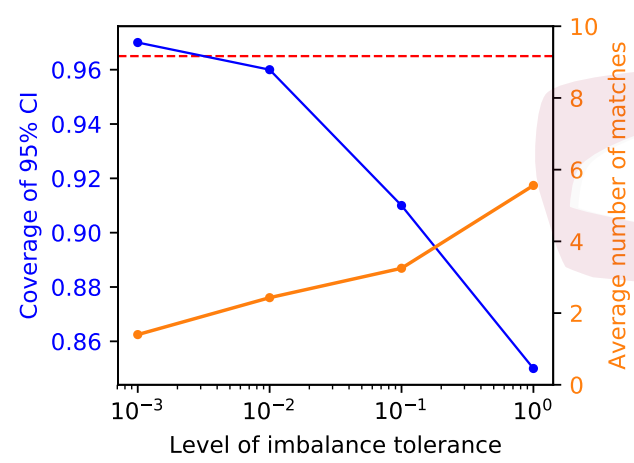

Figure 3: The confidence intervals of matching for balance achieve nominal coverage when the level of imbalance tolerance $\delta$ is small. Increasing $\delta$ trades variance for bias but can degrade the coverage probabilities of the corresponding confidence intervals. In contrast, AIPW (the dashed red line) achieves close-to-nomimal coverage.

An interesting direction for future research is to use a larger value of $\delta$ for estimation, and a smaller value for inference, such that confidence intervals are not necessarily centered at the point estimate. This would be analogous to what is sometimes done in the analysis of regression discontinuity designs (Calonico et al., 2014). In general, how to select the parameter $\delta$ is an open question in causal inference. In the context of weighting, two proposals are provided by Zhao (2019) and Wang \& Zubizarreta (2020), where $\delta$ is selected to generalize covariate balance across cross-validation and bootstrap samples, respectively. 


\section{Concluding Remarks}

We have analyzed a recent class of matching methods that targets aggregate covariate balance. After all, covariate balance is the main diagnostic that investigators carry out in practice. As discussed, matching for balance does not preclude also finding close unit matches, because matching for balance can be followed by matching for homogeneity in order to minimize covariate distances between matched units, while preserving aggregate covariate balance (see Zubizarreta et al. 2014).

Under suitable conditions, we have shown that this class of matching methods yields a simple difference-in-means estimator that is asymptotically optimal: it is $\sqrt{n}$-consistent, asymptotically normal, and semiparametrically efficient. As discussed in the simulation study section, these conditions can be stringent in practice because they require the imbalance tolerance $\delta$ to decrease as the sample size increases and there needs to exist a matching solution for such values of $\delta$. These results complement the fundamental results by Abadie \& Imbens (2006), who showed that a similar estimator is not in general $\sqrt{n}$-consistent for nearest neighbor matching with replacement.

Matching for balance exemplifies how tools from modern optimization 
(e.g., Jünger et al. 2009 and Bixby 2012) can play a central role in the

design of observational studies in general (e.g., Rosenbaum 2002 and Imbens \& Rubin 2015) and in matching for covariate balance in particular (e.g., Zubizarreta et al. 2013 and Keele et al. 2015). A natural future research direction is to augment matching for balance as in doubly robust estimators (Robins et al. 1994; see also Rubin 1979; Abadie \& Imbens 2011; Athey et al. 2018 for related approaches). Another promising direction is to build on the methods described in Rosenbaum (2017) on evidence factors and sensitivity analysis for interpretable analyses of matched observational studies.

\section{Affiliations and acknowledgements}

Yixin Wang, Department of Statistics, University of Michigan, yixinw@ umich . edu; Jose Zubizarreta, Departments of Health Care Policy, Biostatistics, and Statistics, and CAUSALab, Harvard University, zubizarreta@ harvard.edu. For helpful comments and suggestions, we thank Eric Cohn, Bijan Niknam, the editors, and three anonymous reviewers. This work was supported through a Patient-Centered Outcomes Research Institute (PCORI) Project Program Award (ME-2019C1-16172) and grants from the Alfred P. Sloan Foundation (G-2018-10118, G-2020-13946). 


\section{REFERENCES}

\section{References}

Abadie, A. \& Imbens, G. W. (2006). Large sample properties of matching estimators for average treatment effects. Econometrica, 74(1), 235-267.

Abadie, A. \& Imbens, G. W. (2011). Bias-corrected matching estimators for average treatment effects. J Bus Econ Stat, 29(1), 1-11.

Abadie, A. \& Imbens, G. W. (2012). A martingale representation for matching estimators. J Am Stat Assoc, 107(498), 833-843.

Abadie, A. \& Imbens, G. W. (2016). Matching on the estimated propensity score. Econometrica, 84(2), 781-807.

Athey, S., Imbens, G. W., \& Wager, S. (2018). Approximate residual balancing: debiased inference of average treatment effects in high dimensions. J R Stat Soc B, 80(4), 597-623.

Baiocchi, M., Small, D. S., Lorch, S., \& Rosenbaum, P. R. (2010). Building a stronger instrument in an observational study of perinatal care for premature infants. J Am Stat Assoc, 105(492), 1285-1296.

Belloni, A., Chernozhukov, V., Chetverikov, D., \& Kato, K. (2015). Some 


\section{REFERENCES}

new asymptotic theory for least squares series: Pointwise and uniform results. J Econom, 186(2), 345-366.

Bixby, R. E. (2012). A brief history of linear and mixed-integer programming computation. Documenta Mathematica, (pp. 107-121).

Calonico, S., Cattaneo, M. D., \& Titiunik, R. (2014). Robust nonparametric confidence intervals for regression-discontinuity designs. Econometrica, $82(6), 2295-2326$.

Chan, K. C. G., Yam, S. C. P., \& Zhang, Z. (2016). Globally efficient nonparametric inference of average treatment effects by empirical balancing calibration weighting. J R Stat Soc B, 78(3), 673-700.

Chen, X. (2007). Large sample sieve estimation of semi-nonparametric models. Handbook of Econometrics, 6, 5549-5632.

Crump, R. K., Hotz, V. J., Imbens, G. W., \& Mitnik, O. A. (2009). Dealing with limited overlap in estimation of average treatment effects. Biometrika, 96(1), 187-199.

Diamond, A. \& Sekhon, J. S. (2013). Genetic matching for estimating causal effects: A general multivariate matching method for achieving balance in observational studies. Rev Econ Stat, 95(3), 932-945. 


\section{REFERENCES}

Fan, J., Imai, K., Liu, H., Ning, Y., \& Yang, X. (2016). Improving covariate balancing propensity score: A doubly robust and efficient approach.

Fogarty, C. B., Mikkelsen, M. E., Gaieski, D. F., \& Small, D. S. (2016). Discrete optimization for interpretable study populations and randomization inference in an observational study of severe sepsis mortality. $J$ Am Stat Assoc, 111(514), 447-458.

Hainmueller, J. (2012). Entropy balancing for causal effects: a multivariate reweighting method to produce balanced samples in observational studies. Polit Anal, 20(1), 25-46.

Hansen, B. B. (2004). Full matching in an observational study of coaching for the SAT. J Am Stat Assoc, 99(467), 609-618.

Hirshberg, D. A. \& Wager, S. (2018). Augmented minimax linear estimation. arXiv preprint arXiv:1712.00038.

Horowitz, J. L., Mammen, E., et al. (2004). Nonparametric estimation of an additive model with a link function. Ann Stat, 32(6), 2412-2443.

Imai, K. \& Ratkovic, M. (2014). Covariate balancing propensity score. J R Stat Soc B, 76(1), 243-263. 


\section{REFERENCES}

Imbens, G. W. \& Rubin, D. B. (2015). Causal inference in statistics, social, and biomedical sciences. Cambridge University Press.

Imbens, G. W. \& Wooldridge, J. M. (2009). Recent developments in the econometrics of program evaluation. J Econ Lit, 47(1), 5-86.

Jünger, M., Liebling, T. M., et al. (2009). 50 Years of integer programming 1958-2008: From the early years to the state-of-the-art. Springer.

Kallus, N. (2020). Generalized optimal matching methods for causal inference. J Mach Learn Res, 21(62), 1-54.

Keele, L., Titiunik, R., \& Zubizarreta, J. R. (2015). Enhancing a geographic regression discontinuity design through matching to estimate the effect of ballot initiatives on voter turnout. J R Stat Soc A, 178, 223-239.

Newey, W. K. (1997). Convergence rates and asymptotic normality for series estimators. J Econom, 79(1), 147-168.

Neyman, J. $(1923,1990)$. On the application of probability theory to agricultural experiments. Stat Sci, 5(5), 463-480.

Nikolaev, A. G., Jacobson, S. H., Cho, W. K. T., Sauppe, J. J., \& Sewell, E. C. (2013). Balance optimization subset selection: an alternative ap- 


\section{REFERENCES}

proach for causal inference with observational data. Oper Res, 61(2), $398-412$.

Pimentel, S. D., Kelz, R. R., Silber, J. H., \& Rosenbaum, P. R. (2015). Large, sparse optimal matching with refined covariate balance in an observational study of the health outcomes produced by new surgeons. $J$ Am Stat Assoc, 110(510), 515-527.

Redding, S. J. \& Sturm, D. M. (2008). The costs of remoteness: Evidence from german division and reunification. Am Econ Rev, 98(5), 1766-97.

Robins, J. M. \& Ritov, Y. (1997). Toward a curse of dimensionality appropriate (coda) asymptotic theory for semi-parametric models. Stat Med, 16(3), 285-319.

Robins, J. M., Rotnitzky, A., \& Zhao, L. P. (1994). Estimation of regression coefficients when some regressors are not always observed. J Am Stat Assoc, 89(427), 846-866.

Rosenbaum, P. R. (1989). Optimal matching for observational studies. $J$ Am Stat Assoc, 84, 1024-1032.

Rosenbaum, P. R. (2002). Observational studies. Springer. 


\section{REFERENCES}

Rosenbaum, P. R. (2005). Heterogeneity and causality: unit heterogeneity and design sensitivity in observational studies. Am Stat, 59(2), 147-152.

Rosenbaum, P. R. (2010). Design of observational studies. Springer.

Rosenbaum, P. R. (2017). Observation and experiment: an introduction to causal inference. Harvard University Press.

Rosenbaum, P. R. \& Rubin, D. B. (1983). The central role of the propensity score in observational studies for causal effects. Biometrika, 70(1), 41-55.

Rubin, D. B. (1973). Matching to remove bias in observational studies. Biometrics, 29, 159-183.

Rubin, D. B. (1974). Estimating causal effects of treatments in randomized and nonrandomized studies. J Educ Psychol, 66(5), 688.

Rubin, D. B. (1979). Using multivariate matched sampling and regression adjustment to control bias in observational studies. J Am Stat Assoc, 74, $318-328$

Rubin, D. B. (1980). Randomization analysis of experimental data: the fisher randomization test comment. J Am Stat Assoc, 75(371), 591-593. 


\section{REFERENCES}

Van Der Vaart, A. W. \& Wellner, J. A. (1996). Weak convergence. In Weak Convergence and Empirical Processes (pp. 16-28). Springer.

Visconti, G. \& Zubizarreta, J. R. (2018). Handling limited overlap in observational studies with cardinality matching. Obs Stud, 4, 217-249.

Wang, Y. \& Zubizarreta, J. R. (2020). Minimal dispersion approximately balancing weights: asymptotic properties and practical considerations. Biometrika, 107(1), 93-105.

Zhao, Q. (2019). Covariate balancing propensity score by tailored loss functions. Ann Stat, (pp. in press).

Zhao, Q. \& Percival, D. (2017). Entropy balancing is doubly robust. J Causal Inference, 5(1).

Zubizarreta, J. R. (2012). Using mixed integer programming for matching in an observational study of kidney failure after surgery. J Am Stat Assoc, 107(500), 1360-1371.

Zubizarreta, J. R. (2015). Stable weights that balance covariates for estimation with incomplete outcome data. J Am Stat Assoc, 110(511), 910-922. 
Zubizarreta, J. R., Paredes, R. D., \& Rosenbaum, P. R. (2014). Matching for balance, pairing for heterogeneity in an observational study of the effectiveness of for-profit and not-for-profit high schools in chile. Ann Appl Stat, 8(1), 204-231.

Zubizarreta, J. R., Small, D. S., Goyal, N. K., Lorch, S. A., \& Rosenbaum, P. R. (2013). Stronger instruments via integer programming in an observational study of late preterm birth outcomes. Ann Appl Stat, 7, 25-50. 


\section{Supplementary Materials}

\section{A. Proof of Theorem 3.1}

We first revisit the notation. We consider $n$ units randomly drawn from a population. For each unit $i$, we observe its treatment indicator $Z_{i}$, its realized outcome $Y_{i}$, and its pretreatment covariates $X_{i}$. Given the covariate vectors $X_{i}, i=1, \ldots, n$, we consider a vector of $K$ functions of the covariate vector $B(x)=\left(B_{1}(x), \ldots, B_{K}(x)\right)$.

We are interested in estimating the $\operatorname{ATE} \mu=\mathbb{E}\left[Y_{i}(1)-Y_{i}(0)\right]$. Let $m_{i j}$ be a binary indicator of whether unit $i$ is matched with unit $j, i, j=1, \ldots, n$. The general form of the matching for ATE is

$\max . \quad M$

s.t. $\quad m_{i j} \in\{0,1\}, \quad i, j=1, \ldots, n$,

$$
\sum_{j=1}^{n}\left(1-Z_{j}\right) m_{i j}=M, \quad \forall i \in\left\{i: Z_{i}=1\right\},
$$

$$
\begin{aligned}
& \sum_{i=1}^{n} Z_{i} m_{i j}=M, \quad \forall i \in\left\{j: Z_{j}=0\right\} \\
& \sum_{i=1}^{n} \sum_{j=1}^{n} Z_{i} Z_{j} m_{i j}=\sum_{i=1}^{n} \sum_{j=1}^{n}\left(1-Z_{i}\right)\left(1-Z_{j}\right) m_{i j}=0,
\end{aligned}
$$




$$
\begin{aligned}
& \left|\sum_{i=1}^{n} \sum_{j=1}^{n} \frac{Z_{i}\left(1-Z_{j}\right) m_{i j}\left[B_{k}\left(X_{i}\right)-B_{k}\left(X_{j}\right)\right]}{\sum_{i=1}^{n} \sum_{j=1}^{n} Z_{i}\left(1-Z_{j}\right) m_{i j}}\right|<\delta_{k}, \quad k=1, \ldots, K, \\
& \left|\sum_{i=1}^{n} \sum_{j=1}^{n} \frac{\left(1-Z_{i}\right) Z_{j} m_{i j}\left[B_{k}\left(X_{i}\right)-B_{k}\left(X_{j}\right)\right]}{\sum_{i=1}^{n} \sum_{j=1}^{n}\left(1-Z_{i}\right) Z_{j} m_{i j}}\right|<\delta_{k}, \quad k=1, \ldots, K,
\end{aligned}
$$

To estimate the ATE, we compute the difference between the mean outcome of the treated units and that of the matched control units:

$$
\begin{aligned}
\hat{\mu}:=\frac{1}{n}\left[\sum_{i=1}^{n} Z_{i}\left(Y_{i}-\frac{\sum_{j=1}^{m}\left(1-Z_{j}\right) m_{i j} Y_{j}}{\sum_{j=1}^{m}\left(1-Z_{j}\right) m_{i j}}\right)\right. \\
\left.\quad+\sum_{i=1}^{n}\left(1-Z_{i}\right)\left(\frac{\sum_{j=1}^{m} Z_{j} m_{i j} Y_{j}}{\sum_{j=1}^{m} Z_{j} m_{i j}}-Y_{i}\right)\right] .
\end{aligned}
$$

We will give conditions such that the ATE estimator is consistent and asymptotically normal and semiparametric efficient. The asymptotics for the ATE estimator from its corresponding optimization problem can be proved similarly.

The asymptotic properties of $\hat{\mu}$ mainly depends on two of the conditions in the optimization problem, namely Equation (A.3) and Equation A.6).

We first rewrite the ATE estimator (Equation (A.8) as a weighting 
estimator:

$$
\begin{aligned}
\hat{\mu}=\frac{1}{n}\left[\sum_{i=1}^{n} Z_{i} Y_{i}\right. & +\sum_{j=1}^{n} \frac{\sum_{i=1}^{n}\left(1-Z_{i}\right) m_{i j}}{M} Z_{j} Y_{j} \\
& \left.-\sum_{i=1}^{n}\left(1-Z_{i}\right) Y_{i}-\sum_{j=1}^{n} \frac{\sum_{i=1}^{n} Z_{i} m_{i j}}{M}\left(1-Z_{j}\right) Y_{j}\right] .
\end{aligned}
$$

We can similarly rewrite the balancing conditions (Equation $(\mathrm{A} .6)$ and Equation (A.7) as

$$
\begin{array}{r}
\frac{1}{\sum_{i=1}^{n} Z_{i}}\left|\sum_{i=1}^{n} Z_{i} B_{k}\left(X_{i}\right)-\sum_{j=1}^{n} \frac{\sum_{i=1}^{n} Z_{i} m_{i j}}{M}\left(1-Z_{j}\right) B_{k}\left(X_{j}\right)\right|<\delta_{k}, \\
\frac{1}{\sum_{i=1}^{n}\left(1-Z_{i}\right)}\left|\sum_{i=1}^{n}\left(1-Z_{i}\right) B_{k}\left(X_{i}\right)-\sum_{j=1}^{n} \frac{\sum_{i=1}^{n}\left(1-Z_{i}\right) m_{i j}}{M} Z_{j} B_{k}\left(X_{j}\right)\right|<\delta_{k} .
\end{array}
$$

Rewriting the difference-in-means estimator and the balancing conditions implies the following weights assigned to each unit. These implied weights are functions of the covariates,

$$
w\left(X_{j}\right)= \begin{cases}\frac{1}{n}+\frac{\sum_{i=1}^{n}\left(1-Z_{i}\right) m_{i j}}{n M} & \text { if } Z_{j}=1, \\ \frac{1}{n}+\frac{\sum_{i=1}^{n} Z_{i} m_{i j}}{n M} & \text { if } Z_{j}=0 .\end{cases}
$$


If two subjects $j, j^{\prime}$ receive the same treatment assignment $Z_{j}=Z_{j^{\prime}}$ and share the same covariates $X_{j^{\prime}}=X_{j}$ but different weights $w\left(X_{j^{\prime}}\right) \neq w\left(X_{j}\right)$, we define the weights as an average

$$
w\left(X_{j}\right)=\frac{1}{2}\left(w\left(X_{j}\right)+w\left(X_{j^{\prime}}\right)\right) .
$$

Formulating a weight function this way does not affect the value of either $\hat{\mu}$ or the balancing conditions.

For technical convenience, we also assume that $w(x)$ is a piecewise constant function in $x$ with jumps of size at most $O_{p}\left(K\left((\log K) / n+K^{-r_{\pi}}\right) / n\right)$. More technically, there exists $\delta_{0}>0$ such that

$$
\left|Z_{i} w\left(X_{i}\right)-Z_{j} w\left(X_{j}\right)\right|<O_{p}\left(K\left((\log K) / n+K^{-r_{\pi}}\right) / n\right)
$$

and

$$
\left|\left(1-Z_{i}\right) w\left(X_{i}\right)-\left(1-Z_{j}\right) w\left(X_{j}\right)\right|<O_{p}\left(K\left((\log K) / n+K^{-r_{\pi}}\right) / n\right)
$$

for any $\left|X_{i}-X_{j}\right| \leq \delta_{0}$. This assumption roughly implies that units with similar covariates are matched for a similar number of times. Under this 
assumption, the function $w(x)$ can be uniformly approximated by an arbitrarily smooth function up to error $O_{p}\left(K\left((\log K) / n+K^{-r_{\pi}}\right) / n\right)$ due to the compactness of $\mathcal{X}$.

This assumption is satisfied when $1 / M=O_{p}\left(K\left((\log K) / n+K^{-r_{\pi}}\right) / n\right)$, for example, under the overlap condition posited by Proposition 1. If so, it suffices to guarantee that there exists $\delta_{0}>0$ such that $\mid Z_{i} w\left(X_{i}\right)-$ $Z_{j} w\left(X_{j}\right) \mid<1 / M$ and $\left|\left(1-Z_{i}\right) w\left(X_{i}\right)-\left(1-Z_{j}\right) w\left(X_{j}\right)\right|<1 / M$ for any $\left|X_{i}-X_{j}\right| \leq \delta_{0}$. In other words, if two units have similar covariates, then the number of times of each of them being matched can differ by at most one. We can ensure this condition by considering weights obtained by first solving a minimization problem

$$
\begin{aligned}
\min _{w^{0}(\cdot)} & \sum_{k=1}^{K}\left\|\frac{\sum_{i=1}^{n} Z_{i} B_{k}\left(X_{i}\right)-\sum_{j=1}^{n} w^{0}\left(X_{j}\right) B_{k}\left(X_{j}\right)}{\delta_{k} \sum_{i=1}^{n} Z_{i}}\right\|^{2} \\
& +\sum_{k=1}^{K}\left\|\frac{\sum_{i=1}^{n}\left(1-Z_{i}\right) B_{k}\left(X_{i}\right)-\sum_{j=1}^{n} w^{0}\left(X_{j}\right) B_{k}\left(X_{j}\right)}{\delta_{k} \sum_{i=1}^{n}\left(1-Z_{i}\right)}\right\|^{2},
\end{aligned}
$$

then rounding the solution to the nearest $1 / M$ unit, i.e. $w\left(X_{j}\right)=\frac{\left[w^{0}\left(X_{j}\right) \cdot M\right]}{M}$. We note that the $w(\cdot)$ is a smooth function as a solution to the above minimization problem. Under the conditions of Proposition $1, w(\cdot)$ satisfies the piece-wise constant condition above. 
Rewriting the balancing conditions using the $w\left(X_{j}\right)$ notation, we have

$$
\begin{array}{r}
\frac{1}{\sum_{i=1}^{n} Z_{i}}\left|\sum_{i=1}^{n} Z_{i} B_{k}\left(X_{i}\right)-\sum_{j=1}^{n}\left(n w\left(X_{j}\right)-1\right) \cdot\left(1-Z_{j}\right) B_{k}\left(X_{j}\right)\right|<\delta_{k}, \\
\frac{1}{\sum_{i=1}^{n}\left(1-Z_{i}\right)}\left|\sum_{i=1}^{n}\left(1-Z_{i}\right) B_{k}\left(X_{i}\right)-\sum_{j=1}^{n}\left(n w\left(X_{j}\right)-1\right) \cdot Z_{j} B_{k}\left(X_{j}\right)\right|<\delta_{k} .
\end{array}
$$

We first prove the following lemma for the implied weights of the treated units. An analogous lemma holds for the implied weights of the control units.

Lemma 1. Let $\pi(x)$ be the true propensity score function. Under Assumption 1 and Assumption 2, we have, for all treated units,

1. $\sup _{x \in \mathcal{X}}|n w(x)-1 / \pi(x)|=O_{p}\left(K\left((\log K) / n+K^{-r_{\pi}}\right)\right)=o_{p}(1)$

2. $\|n w(x)-1 / \pi(x)\|_{P, 2}=O_{p}\left(K\left((\log K) / n+K^{-r_{\pi}}\right)\right)=o_{p}(1)$.

Proof. For notation convenience, we define $\mathbf{X}=\left(X_{1}, \ldots, X_{n}\right)$. This leads to $B(\mathbf{X})=\left(B\left(X_{1}\right), \ldots, B\left(X_{n}\right)\right)^{\top}$ and $w(\mathbf{X})=\left(w\left(X_{1}\right), \ldots, w\left(X_{n}\right)\right)^{\top}$.

We then define $\mathbf{X}_{\mathcal{C}}=\left(\left\{X_{i}\right\}_{Z_{i}=0}\right)^{\top}$ as the covariate matrix of the control units. This leads to a similar basis matrix $B\left(\mathbf{X}_{\mathcal{C}}\right)$, that is a $C$ by $K$ matrix, 
where $C=\sum_{j=1}^{n}\left(1-Z_{j}\right)$ is the number of control units, and $K$ is the number of basis functions we match. Similarly define $\mathbf{X}_{\mathcal{T}}=\left(\left\{X_{i}\right\}_{Z_{i}=1}\right)^{\top}$ as the covariate matrix of the treated units. $B\left(\mathbf{X}_{\mathcal{T}}\right)$ as the covariate basis matrix of the treated units with size $T$ by $K$, where $T=\sum_{j=1}^{n} Z_{j}$ is the number of treated units.

Denote $\lambda^{\dagger}=\left[B\left(\mathbf{X}_{\mathcal{T}}\right)^{\top} B\left(\mathbf{X}_{\mathcal{T}}\right)\right]^{-1}\left[B\left(\mathbf{X}_{\mathcal{T}}\right)^{\top} w\left(\mathbf{X}_{\mathcal{T}}\right)\right]$. It is the least square projection of the weights $w\left(\mathbf{X}_{\mathcal{T}}\right)$ onto the space of basis functions $B\left(\mathbf{X}_{\mathcal{T}}\right)$.

Below we prove that $\left\|\lambda^{\dagger}-\lambda_{1 T}^{*}\right\|_{2}=O_{p}\left(K^{1 / 2}\left((\log K) / n+K^{-r_{\pi}}\right) / n\right)$ :

$$
\begin{aligned}
& \left\|\lambda^{\dagger}-\lambda_{1 T}^{*}\right\|_{2} \\
= & \left\|\left[B\left(\mathbf{X}_{\mathcal{T}}\right)^{\top} B\left(\mathbf{X}_{\mathcal{T}}\right)\right]^{-1}\left[B\left(\mathbf{X}_{\mathcal{T}}\right)^{\top} w\left(\mathbf{X}_{\mathcal{T}}\right)\right]-\lambda_{1 T}^{*}\right\|_{2} \\
\leq & \left\|B\left(\mathbf{X}_{\mathcal{T}}\right)^{\top} w\left(\mathbf{X}_{\mathcal{T}}\right)-B\left(\mathbf{X}_{\mathcal{T}}\right)^{\top} B\left(\mathbf{X}_{\mathcal{T}}\right) \lambda_{1}^{*}\right\|_{2}\left\|\left[B\left(\mathbf{X}_{\mathcal{T}}\right)^{\top} B\left(\mathbf{X}_{\mathcal{T}}\right)\right]^{-1}\right\|_{2}
\end{aligned}
$$

The first equality is due to the definition of $\lambda^{\dagger}$. The second inequality is due to the Cauchy-Schwarz inequality.

We first consider the term $\left\|B\left(\mathbf{X}_{\mathcal{T}}\right)^{\top} w\left(\mathbf{X}_{\mathcal{T}}\right)-B\left(\mathbf{X}_{\mathcal{T}}\right)^{\top} B\left(\mathbf{X}_{\mathcal{T}}\right) \lambda_{1}^{*}\right\|_{2}$.

$$
\begin{gathered}
\left\|B\left(\mathbf{X}_{\mathcal{T}}\right)^{\top} w\left(\mathbf{X}_{\mathcal{T}}\right)-B\left(\mathbf{X}_{\mathcal{T}}\right)^{\top} B\left(\mathbf{X}_{\mathcal{T}}\right) \lambda_{1}^{*}\right\|_{2} \\
\leq\left\|B\left(\mathbf{X}_{\mathcal{T}}\right)^{\top} w\left(\mathbf{X}_{\mathcal{T}}\right)-B\left(\mathbf{X}_{\mathcal{T}}\right)^{\top}\left(1 /\left\{n \pi\left(\mathbf{X}_{\mathcal{T}}\right)\right\}\right)\right\|_{2}
\end{gathered}
$$




$$
\begin{aligned}
& +\left\|B\left(\mathbf{X}_{\mathcal{T}}\right)^{\top}\left(1 /\left\{n \pi\left(\mathbf{X}_{\mathcal{T}}\right)\right\}\right)-B\left(\mathbf{X}_{\mathcal{T}}\right)^{\top} B\left(\mathbf{X}_{\mathcal{T}}\right) \lambda_{1}^{*}\right\|_{2} \\
= & \left\|B\left(\mathbf{X}_{\mathcal{T}}\right)^{\top} w\left(\mathbf{X}_{\mathcal{T}}\right)-B\left(\mathbf{X}_{\mathcal{T}}\right)^{\top}\left(1 /\left\{n \pi\left(\mathbf{X}_{\mathcal{T}}\right)\right\}\right)\right\|_{2}+\frac{1}{n}\left\|B\left(\mathbf{X}_{\mathcal{T}}\right)\right\|_{2} \cdot O\left(K^{-r_{\pi}}\right) \\
= & \| B\left(\mathbf{X}_{\mathcal{T}}\right)^{\top} w\left(\mathbf{X}_{\mathcal{T}}\right)-\frac{1}{n} B\left(\mathbf { X } ^ { \top } 1 \| _ { 2 } + \| \frac { 1 } { n } B \left(\mathbf{X}^{\top} 1-B\left(\mathbf{X}_{\mathcal{T}}\right)^{\top}\left(1 /\left\{n \pi\left(\mathbf{X}_{\mathcal{T}}\right)\right\}\right) \|_{2}\right.\right. \\
& +\frac{1}{n}\left\|B\left(\mathbf{X}_{\mathcal{T}}\right)\right\|_{2} \cdot O\left(K^{-r_{\pi}}\right) \\
\leq & \|\delta\|_{2} \frac{\sum_{i=1}^{n}\left(1-Z_{i}\right)}{n}+\left\|\frac{1}{n} \sum_{i=1}^{n} B\left(X_{i}\right)-\frac{1}{n} \sum_{i=1}^{n} \frac{Z_{i}}{\pi\left(X_{i}\right)} B\left(X_{i}\right)\right\|_{2} \\
& +\frac{1}{n}\left\|B\left(\mathbf{X}_{\mathcal{T}}\right)\right\|_{2} \cdot O\left(K^{-r_{\pi}}\right) \\
\leq & \|\delta\|_{2} \frac{\sum_{i=1}^{n}\left(1-Z_{i}\right)}{n}+\left\|\frac{1}{n} \sum_{i=1}^{n} \frac{\pi\left(X_{i}\right)-Z_{i}}{\pi\left(X_{i}\right)} B\left(X_{i}\right)\right\|_{2}+O\left(K^{1 / 2-r_{\pi}}\right) \\
\leq & \|\delta\|_{2}+\left\|\frac{1}{n} \sum_{i=1}^{n} \frac{\pi\left(X_{i}\right)-Z_{i}}{\pi\left(X_{i}\right)} B\left(X_{i}\right)\right\|_{2}+O\left(K^{1 / 2-r_{\pi}}\right) .
\end{aligned}
$$

We next bound the middle term $\left\|\frac{1}{n} \sum_{i=1}^{n} \frac{\pi\left(X_{i}\right)-Z_{i}}{\pi\left(X_{i}\right)} B\left(X_{i}\right)\right\|_{2}$ by Bernstein's inequality. Recall that the Bernstein's inequality for random matrices (Tropp et al., 2015) says, let $\left\{W_{k}\right\}$ be a sequence of independent random matrices with dimensions $d_{1} \times d_{2}$. Assume that $\mathbb{E} W_{k}=0$ and $\left\|W_{k}\right\|_{2} \leq R_{n}$ almost surely. Define

$$
\sigma_{n}^{2}=\max \left\{\left\|\sum_{k=1}^{n} \mathbb{E}\left(W_{k} W_{k}^{\top}\right)\right\|_{2},\left\|\sum_{k=1}^{n} \mathbb{E}\left(W_{k}^{\top} W_{k}\right)\right\|_{2}\right\} .
$$


Then for all $t \geq 0$,

$$
P\left(\left\|\sum_{k=1}^{n} W_{k}\right\|_{2} \geq t\right) \leq\left(d_{1}+d_{2}\right) \exp \left(-\frac{t^{2} / 2}{\sigma_{n}^{2}+R_{n} t / 3}\right) .
$$

Therefore, to bound $\left\|1-\frac{1}{n} \sum_{i=1}^{n} \frac{Z_{i}}{\pi\left(X_{i}\right)}\right\|_{2}$, we first show the summand is mean zero

$$
\mathbb{E}\left\{\frac{1}{n} \sum_{i=1}^{n} \frac{\pi\left(X_{i}\right)-Z_{i}}{\pi\left(X_{i}\right)} B\left(X_{i}\right)\right\}=0 .
$$

Furthermore, we have

$$
\begin{aligned}
& \left\|\frac{1}{n} \sum_{i=1}^{n} \frac{\pi\left(X_{i}\right)-Z_{i}}{\pi\left(X_{i}\right)} B\left(X_{i}\right)\right\|_{2} \\
\leq & \frac{1}{n}\left\|\frac{\pi\left(X_{i}\right)-Z_{i}}{\pi\left(X_{i}\right)}\right\|_{2}\left\|B\left(X_{i}\right)\right\|_{2} \\
\leq & \frac{1}{n} \frac{1-\pi\left(X_{i}\right)}{\pi\left(X_{i}\right)} C_{0} K^{1 / 2} \\
\leq & C^{\prime} \frac{K^{1 / 2}}{n},
\end{aligned}
$$

for some constant $C^{\prime}$. The first inequality is due to Cauchy-Schwarz inequality. The second inequality is due to Assumption 1.1 and $E\left(\frac{\pi\left(X_{i}\right)-Z_{i}}{\pi\left(X_{i}\right)}\right)^{2}=$ $\operatorname{Var}\left(\frac{\pi\left(X_{i}\right)-Z_{i}}{\pi\left(X_{i}\right)}\right)=\frac{\left(1-\pi\left(X_{i}\right)\right)\left(\pi\left(X_{i}\right)\right)}{\pi^{2}\left(X_{i}\right)}=\frac{1-\pi\left(X_{i}\right)}{\pi\left(X_{i}\right)}$. The third equality is due to As- 
sumption 2, 1.

Finally, we have

$$
\begin{aligned}
& \left\|\sum_{i=1}^{n} \mathbb{E}\left\{\frac{1}{n^{2}}\left(\frac{\pi\left(X_{i}\right)-Z_{i}}{\pi\left(X_{i}\right)}\right)^{2} B\left(X_{i}\right) B\left(X_{i}\right)^{\top}\right\}\right\|_{2} \\
\leq & \frac{1}{n} \sup _{i}\left(\frac{\pi\left(X_{i}\right)-Z_{i}}{\pi\left(X_{i}\right)}\right)^{2}\left\|E\left\{B\left(X_{i}\right) B\left(X_{i}\right)^{\top}\right\}\right\|_{2} \\
\leq & \frac{C_{1}}{n C_{3}^{2}}=\frac{C^{\prime \prime}}{n}
\end{aligned}
$$

for some constant $C^{\prime \prime}$. The last inequality is due to Assumption 1.2 and Assumption 2.1.

Therefore, by the Bernstein's inequality, we have

$$
P\left(\left\|\frac{1}{n} \sum_{i=1}^{n} \frac{\pi\left(X_{i}\right)-Z_{i}}{\pi\left(X_{i}\right)} B\left(X_{i}\right)\right\|_{2}>t\right) \leq(K+1) \exp \left(-\frac{t^{2} / 2}{\frac{C^{\prime \prime}}{n}+C^{\prime} \frac{K^{1 / 2}}{n} \cdot t / 3}\right) .
$$

When $t=O_{p}\left(K^{1 / 2}(\log K) / n\right)$, then the right side of the inequality going to zero as $n \rightarrow \infty$ and for sufficiently large constants $C^{\prime}$ and $C^{\prime \prime}$. This gives

$$
\left\|\frac{1}{n} \sum_{i=1}^{n} \frac{\pi\left(X_{i}\right)-Z_{i}}{\pi\left(X_{i}\right)} B\left(X_{i}\right)\right\|_{2}=O_{p}\left(K^{1 / 2}(\log K) / n\right) .
$$


Hence, we have

$$
\begin{aligned}
& \left\|B\left(\mathbf{X}_{\mathcal{T}}\right)^{\top} w\left(\mathbf{X}_{\mathcal{T}}\right)-B\left(\mathbf{X}_{\mathcal{T}}\right)^{\top} B\left(\mathbf{X}_{\mathcal{T}}\right) \lambda_{1}^{*}\right\|_{2} \\
\leq & \|\delta\|_{2}+\left\|\frac{1}{n} \sum_{i=1}^{n} \frac{\pi\left(X_{i}\right)-Z_{i}}{\pi\left(X_{i}\right)} B\left(X_{i}\right)\right\|_{2}+O\left(K^{1 / 2-r_{\pi}}\right) \\
\leq & \|\delta\|_{2}+O_{p}\left(K^{1 / 2}(\log K) / n\right)+O\left(K^{1 / 2-r_{\pi}}\right) \\
\leq & \|\delta\|_{2}+O_{p}\left(K^{1 / 2}\left((\log K) / n+K^{-r_{\pi}}\right)\right. \\
\leq & O_{p}\left(K^{1 / 2}\left((\log K) / n+K^{-r_{\pi}}\right) .\right.
\end{aligned}
$$

Finally, this gives

$$
\begin{aligned}
& \left\|\left[B\left(\mathbf{X}_{\mathcal{T}}\right)^{\top} B\left(\mathbf{X}_{\mathcal{T}}\right)\right]^{-1}\left[B\left(\mathbf{X}_{\mathcal{T}}\right)^{\top} w\left(\mathbf{X}_{\mathcal{T}}\right)\right]-\lambda_{1 T}^{*}\right\|_{2} \\
\leq & \left\|\left[B\left(\mathbf{X}_{\mathcal{T}}\right)^{\top} w\left(\mathbf{X}_{\mathcal{T}}\right)\right]-B\left(\mathbf{X}_{\mathcal{T}}\right)^{\top} B\left(\mathbf{X}_{\mathcal{T}}\right) \lambda_{1}^{*}\right\|_{2}\left\|\left[B\left(\mathbf{X}_{\mathcal{T}}\right)^{\top} B\left(\mathbf{X}_{\mathcal{T}}\right)\right]^{-1}\right\|_{2} \\
\leq & O_{p}\left(K^{1 / 2}\left((\log K) / n+K^{-r_{\pi}}\right) \cdot 2 /\left(C_{2} n\right)\right. \\
= & O_{p}\left(K^{1 / 2}\left((\log K) / n+K^{-r_{\pi}}\right) / n\right),
\end{aligned}
$$

where the second inequality is due to the following calucation

$$
\lambda_{\min }\left(B\left(\mathbf{X}_{\mathcal{T}}\right)^{\top} B\left(\mathbf{X}_{\mathcal{T}}\right) / \sum_{i=1}^{n} Z_{i}\right)
$$




$$
\begin{aligned}
& \geq \lambda_{\min }\left(\mathbb{E}\left[B\left(X_{i}\right)^{\top} B\left(X_{i}\right)\right]\right)-\left\|B\left(\mathbf{X}_{\mathcal{T}}\right)^{\top} B\left(\mathbf{X}_{\mathcal{T}}\right) / \sum_{i=1}^{n} Z_{i}-\mathbb{E}\left[B\left(X_{i}\right)^{\top} B\left(X_{i}\right)\right]\right\|_{2} \\
& \geq C_{2}-\left\|B\left(\mathbf{X}_{\mathcal{T}}\right)^{\top} B\left(\mathbf{X}_{\mathcal{T}}\right) / \sum_{i=1}^{n} Z_{i}-\mathbb{E}\left[B\left(X_{i}\right)^{\top} B\left(X_{i}\right)\right]\right\|_{2} \\
& \geq C_{2} / 2
\end{aligned}
$$

for sufficiently large $n$. The first inequality is due to the Weyl inequality. The second inequality is due to Assumption 1. The third inequality is due to $\left\|B\left(\mathbf{X}_{\mathcal{T}}\right)^{\top} B\left(\mathbf{X}_{\mathcal{T}}\right) / \sum_{i=1}^{n} Z_{i}-\mathbb{E}\left[B\left(X_{i}\right)^{\top} B\left(X_{i}\right)\right]\right\|_{2}=o_{p}(1)$ from Lemma D.4 of Fan et al. (2016).

To conclude the proof, we have

$$
\begin{aligned}
& \sup _{x \in \mathcal{X}}\left|n w(x)-\frac{1}{\pi(x)}\right| \\
\leq & \sup _{x \in \mathcal{X}}\left|n w(x)-n B(x) \lambda^{\dagger}\right|+\sup _{x \in \mathcal{X}}\left|n B(x) \lambda^{\dagger}-n B(x) \lambda_{1 T}^{*}\right|+\left|n B(x) \lambda_{1 T}^{*}-\frac{1}{\pi(x)}\right| \\
= & O_{p}\left(K\left((\log K) / n+K^{-r_{\pi}}\right)\right)+O_{p}\left(K^{1 / 2}\left((\log K) / n+K^{-r_{\pi}}\right) / n\right) \cdot O_{p}\left(K^{1 / 2} n\right) \\
& +o_{p}\left(K^{-r_{\pi}}\right) \\
= & O_{p}\left(K\left((\log K) / n+K^{-r_{\pi}}\right)\right)+o_{p}\left(K^{-r_{\pi}}\right) \\
= & O_{p}\left(K\left((\log K) / n+K^{-r_{\pi}}\right)\right) \\
= & O_{p}(1) .
\end{aligned}
$$


The first inequality is due the triangle inequality. The second equality is due to the piece-wise constant condition on $w(\cdot)$ and Assumption 1.1. The last equality is due to Assumption 4.1.

Similarly, we have

$$
\begin{aligned}
& \left\|n w(x)-\frac{1}{1-\pi(x)}\right\|_{2} \\
\leq & O_{p}\left(K^{1 / 2}\left((\log K) / n+K^{-r_{\pi}}\right) / n\right) \cdot O_{p}\left(K^{1 / 2} n\right)+o_{p}\left(K^{-r_{\pi}}\right) \\
= & O_{p}\left(K\left((\log K) / n+K^{-r_{\pi}}\right)\right) \\
= & o_{p}(1) .
\end{aligned}
$$

Analogously, we can prove a similar lemma for the weights of the control units.

Lemma 2. Let $\pi(x)$ be the true propensity score function. Under Assumption 1 and Assumption 2, we have, for all control units,

1. $\sup _{x \in \mathcal{X}}|n w(x)-1 /(1-\pi(x))|=O_{p}\left(K\left((\log K) / n+K^{-r_{\pi}}\right)\right)=o_{p}(1)$,

2. $\|n w(x)-1 /(1-\pi(x))\|_{P, 2}=O_{p}\left(K\left((\log K) / n+K^{-r_{\pi}}\right)\right)=o_{p}(1)$.

Building on Lemma 1 and Lemma 2, we then establish the asymptotic normality and semiparametric efficiency for the ATE estimator Theorem 3.1. 
Proof. The proof utilizes empirical processes techniques as in Fan et al. (2016).

We first decompose $\hat{\mu}-\mu$ into a main term and a few residual terms:

$$
\begin{aligned}
& \hat{\mu}-\mu \\
= & \sum_{i=1}^{n} Z_{i} w\left(X_{i}\right) Y_{i}-\sum_{i=1}^{n}\left(1-Z_{i}\right) w\left(X_{i}\right) Y_{i}-\left(E\left\{Y_{i}(1)\right\}-E\left\{Y_{i}(0)\right\}\right) \\
= & \left(\sum_{i=1}^{n} Z_{i} w\left(X_{i}\right) Y_{i}-E\left\{Y_{i}(1)\right\}\right)-\left(\sum_{i=1}^{n}\left(1-Z_{i}\right) w\left(X_{i}\right) Y_{i}-E\left\{Y_{i}(0)\right\}\right) \\
= & \frac{1}{n} \sum_{i=1}^{n}\left[\frac{Z_{i}}{\pi\left(X_{i}\right)}\left(Y_{i}-Y_{1}\left(X_{i}\right)\right)-\frac{1-Z_{i}}{1-\pi\left(X_{i}\right)}\left(Y_{i}-Y_{0}\left(X_{i}\right)\right)+\left(Y_{1}\left(X_{i}\right)-Y_{0}\left(X_{i}\right)\right)-\mu\right] \\
& +\sum_{i=1}^{n}\left(w\left(X_{i}\right)-\frac{1}{n\left(1-\pi\left(X_{i}\right)\right)}\right)\left(1-Z_{i}\right)\left(Y_{i}-Y_{0}\left(X_{i}\right)\right) \\
& +\sum_{i=1}^{n}\left(w\left(X_{i}\right)-\frac{1}{n \pi\left(X_{i}\right)}\right) Z_{i}\left(Y_{i}-Y_{1}\left(X_{i}\right)\right) \\
& +\sum_{i=1}^{n}\left(w\left(X_{i}\right)\left(1-Z_{i}\right)-\frac{1}{n}\right)\left(Y_{0}\left(X_{i}\right)-B\left(X_{i}\right)^{\top} \lambda_{C}^{*}\right) \\
& +\sum_{i=1}^{n}\left(w\left(X_{i}\right) Z_{i}-\frac{1}{n}\right)\left(Y_{1}\left(X_{i}\right)-B\left(X_{i}\right)^{\top} \lambda_{T}^{*}\right) \\
& +\sum_{i=1}^{n}\left(w\left(X_{i}\right)\left(1-Z_{i}\right)-\frac{1}{n}\right)\left(B\left(X_{i}\right)^{\top} \lambda_{2 C}^{*}\right) \\
& +\sum_{i=1}^{n}\left(w\left(X_{i}\right) Z_{i}-\frac{1}{n}\right)\left(B\left(X_{i}\right)^{\top} \lambda_{2 T}^{*}\right) \\
= & \frac{1}{n} \sum_{i=1}^{n} S_{i}+R_{0 C}+R_{0 T}+R_{1 C}+R_{1 T}+R_{2 C}+R_{2 T},
\end{aligned}
$$


where

$$
\begin{aligned}
S_{i} & =\frac{Z_{i}}{\pi\left(X_{i}\right)}\left(Y_{i}-Y_{1}\left(X_{i}\right)\right)-\frac{1-Z_{i}}{1-\pi\left(X_{i}\right)}\left(Y_{i}-Y_{0}\left(X_{i}\right)\right)+\left(Y_{1}\left(X_{i}\right)-Y_{0}\left(X_{i}\right)\right)-\mu \\
R_{0 C} & =\sum_{i=1}^{n}\left(w\left(X_{i}\right)-\frac{1}{n\left(1-\pi\left(X_{i}\right)\right)}\right)\left(1-Z_{i}\right)\left(Y_{i}-Y_{0}\left(X_{i}\right)\right), \\
R_{0 T} & =\sum_{i=1}^{n}\left(w\left(X_{i}\right)-\frac{1}{n \pi\left(X_{i}\right)}\right) Z_{i}\left(Y_{i}-Y_{1}\left(X_{i}\right)\right), \\
R_{1 C} & =\sum_{i=1}^{n}\left(w\left(X_{i}\right)\left(1-Z_{i}\right)-\frac{1}{n}\right)\left(Y_{0}\left(X_{i}\right)-B\left(X_{i}\right)^{\top} \lambda_{C}^{*}\right), \\
R_{1 T} & =\sum_{i=1}^{n}\left(w\left(X_{i}\right) Z_{i}-\frac{1}{n}\right)\left(Y_{1}\left(X_{i}\right)-B\left(X_{i}\right)^{\top} \lambda_{T}^{*}\right), \\
R_{2 C} & =\sum_{i=1}^{n}\left(w\left(X_{i}\right)\left(1-Z_{i}\right)-\frac{1}{n}\right)\left(B\left(X_{i}\right)^{\top} \lambda_{2 C}^{*}\right), \\
R_{2 T} & =\sum_{i=1}^{n}\left(w\left(X_{i}\right) Z_{i}-\frac{1}{n}\right)\left(B\left(X_{i}\right)^{\top} \lambda_{2 T}^{*}\right) .
\end{aligned}
$$

Below we show $R_{j T}=o_{p}\left(n^{-1 / 2}\right), R_{j C}=o_{p}\left(n^{-1 / 2}\right), 0 \leq j \leq 2$. The conclusion follows from $S_{i}$ taking the same form as the efficient score Hahn, 1998). $\hat{\mu}$ is thus asymptotically normal and semi-parametrically efficient.

Given Lemma 1 and Lemma 2, the rest of the proof repeats the proof structure of Theorem 3 in Wang \& Zubizarreta (2020). They prove a similar consistency and asymptotic normality result for weighting methods. We leverage Lemma 1 and Lemma 2 to adapt their proof to matching for balance. We include the rest of the proof here for completeness. 
The first term we study is $R_{0 C}=\sum_{i=1}^{n}\left(w\left(X_{i}\right)-\frac{1}{n\left(1-\pi\left(X_{i}\right)\right)}\right)\left(1-Z_{i}\right)\left(Y_{i}-\right.$ $\left.Y_{0}\left(X_{i}\right)\right)$. Consider an empirical process $\mathbb{G}_{n}\left(f_{0}\right)=n^{1 / 2}\left(\mathbb{P}_{n}-\mathbb{P}\right) f_{0}(Z, Y, X)$, where $\mathbb{P}_{n}$ stands for the empirical measure and $\mathbb{P}$ stands for the expectation, and

$$
f_{0}(Z, Y, X)=\left(n w(X)-\frac{1}{1-\pi(X)}\right)(1-Z)\left(Y-Y_{0}(X)\right) .
$$

By the unconfoundedness assumption, we have that $\mathbb{P} f_{0}(Z, Y, X)=0$. By Markov's inequality and maximal inequality, we have

$$
\sqrt{n} R_{0} \leq \sup _{f_{0} \in \mathcal{F}} \mathbb{G}_{n}\left(f_{0}\right) \lesssim \mathbb{E} \sup _{f_{0} \in \mathcal{F}} \mathbb{G}_{n}\left(f_{0}\right) \lesssim J_{[]}\left(\left\|F_{0}\right\|_{P, 2}, \mathcal{F}, L_{2}(P)\right),
$$

where the set of functions is $\mathcal{F}=\left\{f_{0}:\|w(\cdot)-1 / n(1-\pi(\cdot))\|_{\infty} \leq \delta\right\}$, where $\|f\|_{\infty}=\sup _{x \in \mathcal{X}}|f(x)|$ and $\delta=C\left(K\left((\log K) / n+K^{-r_{\pi}}\right)\right)$ for some constant $C>0$. The second inequality is due to Markov's inequality. $J_{[]}\left(\left\|F_{0}\right\|_{P, 2}, \mathcal{F}, L_{2}(P)\right.$ is the bracketing integral. $\quad F_{0}:=\delta\left|Y-Y_{0}(X)\right| \gtrsim$ $\left|f_{0}(Z, Y, X)\right|$ is the envelop function. We also have $\left\|F_{0}\right\|_{P, 2}=\left(\mathbb{P} F_{0}^{2}\right)^{1 / 2} \lesssim \delta$ by $\mathbb{E}\left|Y-Y_{0}(X)\right|<\infty$. This construction is due to Lemma 1, where we have

$$
\left\|n w(x)-\frac{1}{1-\pi(x)}\right\|_{2}=O_{p}\left(K\left((\log K) / n+K^{-r_{\pi}}\right)\right)=o_{p}(1) .
$$

We next show $\mathbb{E} \sup _{f_{0} \in \mathcal{F}} \mathbb{G}_{n}\left(f_{0}\right) \rightarrow 0$ as $\delta \rightarrow 0$. We bound $J_{[}\left(\left\|F_{0}\right\|_{P, 2}, \mathcal{F}, L_{2}(P)\right.$ 
by $n_{\square}\left(\varepsilon, \mathcal{F}, L_{2}(P)\right)$ :

$$
J_{[]}\left(\left\|F_{0}\right\|_{P, 2}, \mathcal{F}, L_{2}(P) \lesssim \int_{0}^{\delta} \sqrt{n_{\square}\left(\varepsilon, \mathcal{F}, L_{2}(P)\right)} \mathrm{d} \varepsilon\right.
$$

Then we have

$$
\begin{aligned}
\log n_{\square}\left(\varepsilon, \mathcal{F}, L_{2}(P)\right) & \lesssim \log n_{\square}\left(\varepsilon, \mathcal{F}_{0} \delta, L_{2}(P)\right) \\
& =\log n_{\square}\left(\varepsilon / \delta, \mathcal{F}_{0}, L_{2}(P)\right) \\
& \lesssim \log n_{\square}\left(\varepsilon / \delta, \mathcal{M}, L_{2}(P)\right. \\
& \lesssim(\delta / \varepsilon)^{\left(1 / k_{1}\right)}
\end{aligned}
$$

where we consider a new set of functions $\mathcal{F}_{0}=\left\{f_{0}:\|w(\cdot)-1 / n(1-\pi(\cdot))\|_{\infty} \leq\right.$ $C$ \} for some constant $C>0$. The first inequality is due to $w(\cdot)$ bounded away from 0 and (can always be made) Lipschitz. The last inequality is due to Assumption 3.4 .

Therefore, we have

$$
J_{\square}\left(\left\|F_{0}\right\|_{P, 2}, \mathcal{F}, L_{2}(P) \lesssim \int_{0}^{\delta} \sqrt{\log n_{\square}\left(\varepsilon, \mathcal{F}, L_{2}(P)\right)} d \varepsilon \lesssim \int_{0}^{\delta}(\delta / \varepsilon)^{\left(1 / 2 k_{1}\right)} d \varepsilon .\right.
$$

This goes to 0 as $\delta$ goes to 0 by $2 k_{1}>1$ and the integral converges. 
Thus, this shows that $n^{1 / 2} R_{0 C}=o_{p}(1)$. With the exact same argument, we can also show that $n^{1 / 2} R_{0 T}=o_{p}(1)$.

Next, we consider $R_{1 C}=\sum_{i=1}^{n}\left(w\left(X_{i}\right)\left(1-Z_{i}\right)-\frac{1}{n}\right)\left(Y_{0}\left(X_{i}\right)-B\left(X_{i}\right)^{\top} \lambda_{C}^{*}\right)$. Define the empirical process $\mathbb{G}_{n}\left(f_{1}\right)=n^{1 / 2}\left(\mathbb{P}_{n}-\mathbb{P}\right) f_{1}(Z, X)$, where

$$
f_{1}(Z, X)=(n(1-Z) w(X)-1)\left(Y_{0}(X)-B(X)^{\top} \lambda_{2 C}^{*}\right) .
$$

We have

$$
\begin{aligned}
n^{1 / 2} R_{1} & =\mathbb{G}_{n}\left(f_{1}\right)+n^{1 / 2} \mathbb{P} f_{1}(Z, X) \\
& \leq \sup _{f_{1} \in \mathcal{F}_{1}} \mathbb{G}_{n}\left(f_{1}\right)+n^{1 / 2} \sup _{f_{1} \in \mathcal{F}_{1}} \mathbb{P} f_{1},
\end{aligned}
$$

where $\Delta(X):=Y_{0}\left(X_{i}\right)-B\left(X_{i}\right)^{\top} \lambda_{C}^{*}, \mathcal{F}_{1}=\left\{f_{1}:\|w(\cdot)-1 / n(1-\pi(\cdot))\|_{P, 2} \leq\right.$ $\left.\delta_{1},\|\Delta\|_{\infty} \leq \delta_{2}\right\}, \delta_{1}=C\left(K\left((\log K) / n+K^{-r_{\pi}}\right)\right)$, and $\delta_{2}=C K^{-r_{y}}$ for some constant $C>0$. This construction is due to Lemma 2,2 and Assumption 3.3.

Similar to characterizing $R_{0 C}$, we have

$$
\sup _{f_{1} \in \mathcal{F}_{1}} \mathbb{G}_{n}\left(f_{1}\right) \lesssim \mathbb{E} \sup _{f_{1} \in \mathcal{F}_{1}} \mathbb{G}_{n}\left(f_{1}\right) \lesssim J_{[]}\left(\left\|F_{1}\right\|_{P, 2}, \mathcal{F}, L_{2}(P)\right)
$$


where $F_{1}:=C \delta_{2}$ for some constant $C>0$ so that $\left\|F_{1}\right\|_{P, 2} \lesssim \delta_{2}$. We then bound $J_{\square}\left(\left\|F_{1}\right\|_{P, 2}, \mathcal{F}_{1}, L_{2}(P)\right): J_{\square}\left(\left\|F_{1}\right\|_{P, 2}, \mathcal{F}_{1}, L_{2}(P) \lesssim \int_{0}^{\delta} \sqrt{n_{\square}\left(\varepsilon, \mathcal{F}_{1}, L_{2}(P)\right)} \mathrm{d} \varepsilon\right.$.

Moreover, we have

$$
\begin{aligned}
\log n_{\square}\left(\varepsilon, \mathcal{F}_{1}, L_{2}(P)\right) & \lesssim \log n_{\llbracket}\left(\varepsilon / \delta_{2}, \mathcal{F}_{0}, L_{2}(P)\right) \\
& \lesssim \log n_{\square}\left(\varepsilon / \delta_{2}, G_{10}, L_{2}(P)\right)+\log n_{\square}\left(\varepsilon / \delta_{2}, G_{20}, L_{2}(P)\right) \\
& \lesssim \log n_{\llbracket}\left(\varepsilon / \delta_{2}, \mathcal{M}, L_{2}(P)\right)+\log n_{\square}\left(\varepsilon / \delta_{2}, \mathcal{H}, L_{2}(P)\right) \\
& \lesssim\left(\delta_{1} / \varepsilon\right)^{1 / k_{1}}+\left(\delta_{2} / \varepsilon\right)^{1 / k_{2}},
\end{aligned}
$$

where $\mathcal{F}_{0}=\left\{f_{1}:\|w(\cdot)-1 /(1-\pi(\cdot))\|_{P, 2} \leq C,\|\Delta\|_{P, 2} \leq 1\right\}, \mathcal{G}_{10}=\{m \in$ $\left.\mathcal{M}+1 /(1-\pi(\cdot)):\|w\|_{P, 2} \leq C\right\}, \mathcal{G}_{20}=\left\{\Delta \in \mathcal{H}-B\left(X_{i}\right)^{\top} \lambda_{2 C}^{*}:\|\Delta\|_{P, 2} \leq 1\right\}$.

Therefore we have

$$
J_{\square}\left(\left\|F_{1}\right\|_{P, 2}, \mathcal{F}_{1}, L_{2}(P) \lesssim \int_{0}^{\delta}\left(\delta_{1} / \varepsilon\right)^{\left(1 / 2 k_{1}\right)} d \varepsilon+\int_{0}^{\delta}\left(\delta_{2} / \varepsilon\right)^{\left(1 / 2 k_{2}\right)} d \varepsilon\right.
$$

By $2 k_{1}>1,2 k_{2}>1$ (Assumption 3.4), we have $J_{\square}\left(\left\|f_{1}\right\|_{P, 2}, \mathcal{F}, L_{2}(P)\right)=$ $o(1)$. This gives $\sup _{f_{1} \in \mathcal{F}_{1}} \mathbb{G}_{n}\left(f_{1}\right)=o_{p}(1)$.

Finally we show that $n^{1 / 2} \sup _{f_{1} \in \mathcal{F}_{1}} \mathbb{P} f_{1}=o_{p}(1)$.

$$
\left.n^{1 / 2} \sup _{f_{1} \in \mathcal{F}} \mathbb{P} f_{1}=n^{1 / 2} \sup _{m \in \mathcal{G}_{1}, \Delta \in \mathcal{G}_{2}} \mathbb{E}(n(1-Z) w(X)-Z / \pi(X)) \Delta(X)\right)
$$




$$
\begin{aligned}
& =n^{1 / 2} \sup _{m \in \mathcal{G}_{1}, \Delta \in \mathcal{G}_{2}} \mathbb{E}((n w(X)(1-\pi(X))-1) \Delta(x)) \\
& \lesssim n^{1 / 2} \sup _{m \in \mathcal{G}_{1}}\left\|n w(x)-\frac{1}{1-\pi(x)}\right\|_{P, 2} \sup _{\Delta \in \mathcal{G}_{2}}\|\Delta(x)\|_{P, 2} \\
& \lesssim n^{1 / 2} \delta_{1} \delta_{2}=o_{p}(1),
\end{aligned}
$$

where $\mathcal{G}_{1}=\left\{m \in \mathcal{M}:\|w(\cdot)-1 /(1-\pi(\cdot))\|_{P, 2} \leq \delta_{1}\right\}, \mathcal{G}_{2}=\{\Delta \in \mathcal{H}-$ $\left.B\left(X_{i}\right)^{\top} \lambda_{2 C}^{*}:\|\Delta\|_{\infty} \leq \delta_{2}\right\}$. The last equality is due to assumption $n^{1 / 2} \lesssim$ $K^{r_{\pi}+r_{y}-1 / 2}$.

Therefore, we can conclude $n^{1 / 2} R_{1 C}=o_{p}(1)$. Analogously, we can conclude $n^{1 / 2} R_{1 T}=o_{p}(1)$.

Lastly, we study $R_{2 C}=\sum_{i=1}^{n}\left(w\left(X_{i}\right)\left(1-Z_{i}\right)-\frac{1}{n}\right)\left(B\left(X_{i}\right)^{\top} \lambda_{2 C}^{*}\right)$. We have $R_{2 C}=o_{p}\left(n^{-1 / 2}\right)$ because the covariate balancing condition in the optimization problem and Assumption 4.2. Similarly, we have $R_{2 T}=o_{p}\left(n^{-1 / 2}\right)$.

After establishing consistency and asymptotic normality, we provide an estimator for the asymptotic variance. We construct the variance estimator by approximating the efficient influence function. This construction and the proof mimics the second part of Theorem 3 in Wang \& Zubizarreta (2020).

First recall that the semiparametric efficiency bound for ATE (Hahn, 
1998) is

$$
\begin{aligned}
V_{\text {opt }}:= & \mathbb{E}\left[\frac{\left.\operatorname{var}\left(Y_{i}(1) \mid X_{i}\right)\right)}{\pi\left(X_{i}\right)}+\frac{\left.\operatorname{var}\left(Y_{i}(0) \mid X_{i}\right)\right)}{1-\pi\left(X_{i}\right)}+\left(E\left(Y_{i}(1)-Y_{i}(0) \mid X_{i}\right)-\mu\right)^{2}\right] \\
= & \mathbb{E}\left[\left(\frac{Z_{i} Y_{i}}{\pi\left(X_{i}\right)}-\bar{Y}_{i}(1)\right)-\left(\frac{\left(1-Z_{i}\right) Y_{i}}{1-\pi\left(X_{i}\right)}-\bar{Y}_{i}(0)\right)\right. \\
& \left.-\left(Y_{1}\left(X_{i}\right)\left(\frac{Z_{i}}{\pi\left(X_{i}\right)}-1\right)-Y_{0}\left(X_{i}\right)\left(\frac{1-Z_{i}}{1-\pi\left(X_{i}\right)}-1\right)\right)\right]^{2}
\end{aligned}
$$

To estimate this variance, we consider the following estimator

$$
\begin{aligned}
\hat{V}_{K}= & \sum_{i=1}^{n}\left[Z_{i} w\left(X_{i}\right) Y_{i}-\frac{\sum_{i=1}^{n} Z_{i} w\left(X_{i}\right) Y_{i}}{\sum_{i=1}^{n} Z_{i}}\right. \\
& -\left(1-Z_{i}\right) w\left(X_{i}\right) Y_{i}+\frac{\sum_{i=1}^{n}\left(1-Z_{i}\right) w\left(X_{i}\right) Y_{i}}{\sum_{i=1}^{n}\left(1-Z_{i}\right)} \\
& -\hat{Y}_{T}\left(X_{i}\right) \times\left(Z_{i} w\left(X_{i}\right)-\frac{1}{\sum_{i=1}^{n} Z_{i}}\right) \\
& \left.+\hat{Y}_{C}\left(X_{i}\right) \times\left(\left(1-Z_{i}\right) w\left(X_{i}\right)-\frac{1}{\sum_{i=1}^{n}\left(1-Z_{i}\right)}\right)\right]^{2} .
\end{aligned}
$$

where

$$
\begin{aligned}
& \hat{Y}_{T}\left(X_{i}\right)=B\left(X_{i}\right)^{\top}\left\{\frac{\sum_{i=1}^{n} Z_{i} w\left(X_{i}\right) B\left(X_{i}\right)^{\top} B\left(X_{i}\right)}{\sum_{i=1}^{n} Z_{i}}\right\}^{-1} \\
& \cdot\left\{\frac{\sum_{i=1}^{n} Z_{i} w\left(X_{i}\right) B\left(X_{i}\right)^{\top} Y_{i}}{\sum_{i=1}^{n} Z_{i}}\right\}
\end{aligned}
$$


and

$$
\begin{aligned}
& \hat{Y}_{C}\left(X_{i}\right)=B\left(X_{i}\right)^{\top}\left\{\frac{\sum_{i=1}^{n}\left(1-Z_{i}\right) w\left(X_{i}\right) B\left(X_{i}\right)^{\top} B\left(X_{i}\right)}{\sum_{i=1}^{n}\left(1-Z_{i}\right)}\right\}^{-1} \\
& \cdot\left\{\frac{\sum_{i=1}^{n}\left(1-Z_{i}\right) w\left(X_{i}\right) B\left(X_{i}\right)^{\top} Y_{i}}{\sum_{i=1}^{n}\left(1-Z_{i}\right)}\right\}
\end{aligned}
$$

are least square estimators of $Y_{1}\left(X_{i}\right)$ and $Y_{0}\left(X_{i}\right)$ respectively.

To prove consistency, it is sufficient show that

$$
\begin{aligned}
& \hat{Y}_{T}\left(X_{i}\right)-Y_{1}\left(X_{i}\right) \stackrel{\text { a.s. }}{\rightarrow} 0, \\
& \hat{Y}_{C}\left(X_{i}\right)-Y_{0}\left(X_{i}\right) \stackrel{\text { a.s. }}{\rightarrow} 0,
\end{aligned}
$$

because we have shown that $n w\left(X_{i}\right)$ is consistent for $\pi\left(X_{i}\right)$ and $\frac{\sum_{i=1}^{n} Z_{i} w\left(X_{i}\right) Y_{i}}{\sum_{i=1}^{n} Z_{i}}-$ $\frac{\sum_{i=1}^{n}\left(1-Z_{i}\right) w\left(X_{i}\right) Y_{i}}{\sum_{i=1}^{n}\left(1-Z_{i}\right)}$ is consistent for $\mu$.

Below we assume a stronger smoothness assumption, i.e. $r_{y}>1$.

To prove Equation A.18, we first rewrite $Y_{i}$ as $Y_{1}\left(X_{i}\right)=B\left(X_{i}\right)^{\top} \lambda_{2 T}^{*}+$ $\gamma+\epsilon_{i}$, where $\gamma=O\left(K^{-r_{y}}\right)$ from Assumption 3.3, and $\epsilon_{i}$ is some iid zero mean error with variance $\sigma^{2}=\operatorname{var}\left(Y_{1}\left(X_{i}\right) \mid X_{i}\right)$. Therefore,

$$
\left\{\frac{\sum_{i=1}^{n} Z_{i} w\left(X_{i}\right) B\left(X_{i}\right)^{\top} B\left(X_{i}\right)}{\sum_{i=1}^{n} Z_{i}}\right\}^{-1} \cdot\left\{\frac{\sum_{i=1}^{n} Z_{i} w\left(X_{i}\right) B\left(X_{i}\right)^{\top} Y_{i}}{\sum_{i=1}^{n} Z_{i}}\right\}
$$




$$
\begin{aligned}
= & \left\{\frac{\sum_{i=1}^{n} Z_{i} w\left(X_{i}\right) B\left(X_{i}\right)^{\top} B\left(X_{i}\right)}{\sum_{i=1}^{n} Z_{i}}\right\}^{-1} \cdot\left\{\frac{\sum_{i=1}^{n} Z_{i} w\left(X_{i}\right) B\left(X_{i}\right)^{\top}\left\{B\left(X_{i}\right)^{\top} \lambda_{2}^{*}+\gamma+\epsilon_{i}\right\}}{\sum_{i=1}^{n} Z_{i}}\right\} \\
= & \lambda_{2}^{*}+\left\{\frac{\sum_{i=1}^{n} Z_{i} w\left(X_{i}\right) B\left(X_{i}\right)^{\top} B\left(X_{i}\right)}{\sum_{i=1}^{n} Z_{i}}\right\}^{-1} \cdot\left\{\frac{\sum_{i=1}^{n} Z_{i} w\left(X_{i}\right) B\left(X_{i}\right)^{\top}\left\{B\left(X_{i}\right)^{\top} \gamma\right\}}{\sum_{i=1}^{n} Z_{i}}\right\} \\
& +\left\{\frac{\sum_{i=1}^{n} Z_{i} w\left(X_{i}\right) B\left(X_{i}\right)^{\top} B\left(X_{i}\right)}{\sum_{i=1}^{n} Z_{i}}\right\}^{-1} \cdot\left\{\frac{\sum_{i=1}^{n} Z_{i} w\left(X_{i}\right) B\left(X_{i}\right)^{\top} \epsilon_{i}}{\sum_{i=1}^{n} Z_{i}}\right\} \\
= & \lambda_{2}^{*}+E\left\{Z_{i} w\left(X_{i}\right) B\left(X_{i}\right)^{\top} B\left(X_{i}\right)\right\}^{-1} E\left\{Z_{i} w\left(X_{i}\right) B\left(X_{i}\right)^{\top}\right\}\left\{\gamma+E\left(\epsilon_{i}\right)\right\}+O_{p}\left(n^{-1 / 2}\right) \\
= & \lambda_{2 T}^{*}+O_{p}\left(K^{-r_{y}+1 / 2}\right),
\end{aligned}
$$

where the last equality is due to Assumption 1,4 and Assumption 3.3 and the law of large numbers.

Finally we have

$$
\begin{aligned}
& B\left(X_{i}\right)^{\top}\left\{\frac{\sum_{i=1}^{n} Z_{i} w\left(X_{i}\right) B\left(X_{i}\right)^{\top} B\left(X_{i}\right)}{\sum_{i=1}^{n} Z_{i}}\right\}^{-1} \cdot\left\{\frac{\sum_{i=1}^{n} Z_{i} w\left(X_{i}\right) B\left(X_{i}\right)^{\top} Y_{i}}{\sum_{i=1}^{n} Z_{i}}\right\} \\
= & B\left(X_{i}\right)^{\top} \lambda_{2 T}^{*}+B\left(X_{i}\right) \cdot O_{p}\left(K^{-r_{y}+1 / 2}\right) \\
= & Y_{1}\left(X_{i}\right)+B\left(X_{i}\right) \cdot O_{p}\left(K^{-r_{y}+1 / 2}\right)+O_{p}\left(K^{-r_{y}}\right) \\
= & Y_{1}\left(X_{i}\right)+o_{p}(1)
\end{aligned}
$$

The last equality is due to assumption Assumption 1,4 and the additional assumption $r_{y}>1$.

Equation A.19 can be proved in an analogous way. Hence we estab- 
lished the consistency of $\hat{V}_{K}$.

\section{B. Existence of a solution}

In this section, we provide sufficient conditions that guarantee the existence of a solution to the matching for balance optimization problem. As discussed, if there is no solution for the matching for balance problem, there will likely be no solution that balances covariates also for other matching or weighting methods with positive weights, i.e. that do not extrapolate. Together with Theorem 3.1, this result describes the settings where matching methods are as statistically efficient as weighting methods. Moreover, they are both semiparametrically efficient.

Proposition 1. (Sufficient conditions for the existence of a solution to matching for balance) If there exists a constant $C_{3}>0$ such that $C_{3}<$ $\pi(x)<1-C_{3}$ and

$$
C_{3} \geq \Theta\left(1 /\left((\log K)+n K^{-r_{\pi}}\right)\right)
$$

then matching for balance admits a solution with probability $1-\delta_{0}$ under Assumptions 1 to 4 when $n \geq \log _{1-\rho}\left(\delta_{0} 2^{-K}\right)$ for a constant $\rho \in(0,1)$. (The precise technical definition of the constant $\rho$ is in Appendix $C$ of the 
Supplementary Materials.)

Proposition 1 describes sufficient conditions for a matching-for-balance solution to exist. It roughly requires that the propensity score function should be bounded away from zero and one; it must be at least $\Theta(1 /((\log K)+$ $\left.\left.n K^{-r_{\pi}}\right)\right)$ away. As the number of units $n$ increases, this requirement becomes increasingly weak and a match-for-balance solution exists more likely. Together with Theorem 3.1, Proposition 1 delineates a setting where matching methods can be as efficient as weighting methods despite its integer constraints. In this setting, matching for balance is asymptotically optimal: it is $\sqrt{n}$-consistent, asymptotically normal, and semiparametrically efficient.

The intuition behind Proposition 1 is twofold: (1) the treated and control population are closer if the propensity score is farther from zero and one; (2) if the two populations are closer, it is more likely for a matching solution to exist, i.e. satisfy the covariate balancing constraints in Equations (2.6) and (2.7). Due to this intuition, we posit the overlap condition in Proposition 1 to constrain how far away the two populations can be. It requires the minimum propensity score $C_{3}$ to be larger than $\Theta\left(1 /\left((\log K)+n K^{-r_{\pi}}\right)\right)$. This condition is stronger than what is usually required of the overlap between the treated and the control (e.g., Assumption 2.1). Nevertheless, it 
guarantees the existence and asymptotic optimality of a matching solution. The full proof of Proposition 1 is in Appendix C of the Supplementary Material.

\section{Proof of Proposition 1}

We first define the constant $\rho$ in Proposition 1. The constant $\rho$ is defined as $\rho \triangleq \min _{i} P\left(X \in R_{i} \mid T=0\right)>0$, where $R_{i}, i=1, \ldots, 3^{K}$, are the $3^{K}$ boxes centered at $E\left[B\left(X_{i}\right) \mid Z_{i}=1\right]+\frac{3}{2} \delta \odot b$. The constant $\delta=\left(\delta_{1}, \ldots, \delta_{k}\right)$ is the covariate imbalances allowed in matching for balance (Equations 2.6 and (2.7) ) and $b \in \mathbb{R}^{K}$ is a vector that each entry can be $-1,0,1$.

We will prove the existence of $w_{C}\left(X_{j}\right)$ that solves the optimization problem of matching for balance, where $w_{C}\left(X_{j}\right)=\left(\sum_{i=1}^{n} Z_{i} m_{i j}\right) / M$. The exact same argument can establish the existence of $w_{T}\left(X_{j}\right)$.

The proof proceeds in three steps: (1) Show that a set of $w_{C}\left(X_{i}\right)$ exists that satisfies Equation (2.6), without conforming to the form of Equation 2.10. (2) Show that a set of $w_{C}\left(X_{i}\right)$ exists that satisfies both Equation (2.6) and Equation 2.10). (3) Show that a set of $m_{i j}$ satisfies both Equation (2.6) and Equation (2.10).

The first step follows directly from Lemma 2 of Zhao \& Percival (2017). 
They show that a solution exists for Equation (2.6) with probability $\delta_{0}$ when the number of units satisfy $n \geq \log _{1-\rho}\left(\delta_{0} \cdot 2^{-K}\right)$. We call this solution $w_{C}^{0}\left(X_{i}\right)$.

The second step relies on the following observation: Equation (2.10) amounts to the restriction that we can only approximate $w_{C}^{0}\left(X_{i}\right)$ up to the precision $(M)^{-1}$. The reason is that $w_{C}\left(X_{i}\right)=\left(\sum_{i=1}^{n} Z_{i} m_{i j}\right) / M \in$ $\{k / M, k \in \mathbb{N}\}$; matching restricts both the numerator $\left(\sum_{i=1}^{n} Z_{i} m_{i j}\right)$ and the denominator $M$ of $w_{C}\left(X_{i}\right)$ to be integers.

However, we show that the $w_{C}\left(X_{i}\right)=\left(\sum_{i=1}^{n} Z_{i} m_{i j}\right) / M$ closest to $w_{C}^{0}\left(X_{i}\right)$ still satisfies Equation (2.6). Notice that $\left|w_{C}\left(X_{i}\right)-w_{C}^{0}\left(X_{i}\right)\right|<1 / M$. This implies

$$
\begin{aligned}
& \frac{1}{\sum_{i=1}^{n} Z_{i}}\left|\sum_{i=1}^{n} Z_{i} B_{k}\left(X_{i}\right)-\sum_{j=1}^{n} w_{C}\left(X_{j}\right)\left(1-Z_{j}\right) B_{k}\left(X_{j}\right)\right| \\
\leq & \frac{1}{\sum_{i=1}^{n} Z_{i}}\left|\sum_{i=1}^{n} Z_{i} B_{k}\left(X_{i}\right)-\sum_{j=1}^{n} w_{C}^{0}\left(X_{j}\right)\left(1-Z_{j}\right) B_{k}\left(X_{j}\right)\right| \\
& +\frac{1}{\sum_{i=1}^{n} Z_{i}}\left|\sum_{j=1}^{n} w_{C}^{0}\left(X_{j}\right)\left(1-Z_{j}\right) B_{k}\left(X_{j}\right)-\sum_{j=1}^{n} w_{C}\left(X_{j}\right)\left(1-Z_{j}\right) B_{k}\left(X_{j}\right)\right| \\
\leq & \delta_{k}+\frac{\sum_{i=1}^{n}\left(1-Z_{i}\right)}{\sum_{i=1}^{n} Z_{i}} \frac{1}{M}\left|\sum_{j=1}^{n} B_{k}\left(X_{i}\right)\right| \\
\triangleq & \delta_{k}^{M}
\end{aligned}
$$


Assumption 42 of Theorem 3.1 requires that $\|\delta\|_{2}=O_{p}\left(K^{1 / 2}((\log K) / n+\right.$ $\left.K^{-r_{\pi}}\right)$, where $\delta=\left(\delta_{1}, \ldots, \delta_{K}\right)$. We will show that $\left\|\delta^{M}\right\|_{2}$ also satisfies Assumption 4 2. Therefore, $w_{C}\left(X_{j}\right)$ can also lead to consistent, asymptotically normal, and semiparametrically efficient average treatment effect (ATE) estimators.

We bound the norm of $\left\|\delta^{M}\right\|_{2}$ :

$$
\begin{aligned}
\left\|\delta^{M}\right\|_{2} & \leq\|\delta\|_{2}+\sum_{k=1}^{K}\left[\frac{\sum_{i=1}^{n}\left(1-Z_{i}\right)}{\sum_{i=1}^{n} Z_{i}} \frac{1}{M}\left|\sum_{j=1}^{n} B_{k}\left(X_{i}\right)\right|\right] \\
& \leq\|\delta\|_{2}+\frac{C}{M} \frac{\sum_{i=1}^{n}\left(1-Z_{i}\right)}{\sum_{i=1}^{n} Z_{i}} K^{1 / 2} \\
& =O_{p}\left(K^{1 / 2}\left((\log K) / n+K^{-r_{\pi}}\right) .\right.
\end{aligned}
$$

The last equality is because we can let $M=\Theta\left(\left((\log K) / n+K^{-r_{\pi}}\right)^{-1}\right)$. It is feasible because the number of match required $M$ is smaller than the number of treated units or control units:

$$
\begin{aligned}
M & =\Theta\left(\left((\log K) / n+K^{-r_{\pi}}\right)^{-1}\right) \\
& \leq n C_{3}-\sqrt{\log (1-\rho)+\frac{K \log 2}{n}} \\
& \leq n C_{3}-\sqrt{\left(-\log \delta_{0}\right) /(2 n)} \\
& \leq \min \left(\sum_{i=1}^{n} Z_{i}, \sum_{i=1}^{n} 1-Z_{i}\right)
\end{aligned}
$$


with probability $1-\delta_{0}$. The first inequality is due to Equation (B.1). The second inequality is due to the assumption that $n \geq \log _{1-\rho}\left(\delta_{0} \cdot 2^{-K}\right)$. The third inequality is due to the Hoeffding's inequality.

The third step is to construct a solution $\left\{m_{i j}\right\}_{i, j=1}^{n}$ such that they are consistent with the solution from the second step: $w_{C}\left(X_{j}\right)=\left(\sum_{i=1}^{n} Z_{i} m_{i j}\right) / M$. We first notice that we only need to make sure each treated unit is matched $M$ times. Moreover, for each control unit $j$, it is matched $w_{C}\left(X_{j}\right) \cdot M$ times. Therefore, to construct a solution $m_{i j}$, we match each control unit to the first $w_{C}\left(X_{j}\right) \cdot M$ treated units that have not been matched for $M$ times. More precisely, we start with setting $m_{i j}=0$ for all $i, j$. We then iterate through the set of control units $\left\{i: Z_{i}=0\right\}$. For each $i$, we set $m_{i j}=1$ if $j$ satisfies $\sum_{k=1}^{j} \mathbb{1}\left\{\sum_{j=1}^{n} m_{k j}<M\right\}<M$. Iterating through all the control units leads to a solution $m_{i j}$ that satisfies both Equation 2.6 and Equation 2.10.

\section{References}

Fan, J., Imai, K., Liu, H., Ning, Y., \& Yang, X. (2016). Improving covariate balancing propensity score: A doubly robust and efficient approach.

Hahn, J. (1998). On the role of the propensity score in efficient semi- 


\section{REFERENCES}

parametric estimation of average treatment effects. Econometrica, (pp. $315-331)$.

Tropp, J. A. et al. (2015). An introduction to matrix concentration inequalities. Foundations and Trends(R) in Machine Learning, 8(1-2), 1-230.

Wang, Y. \& Zubizarreta, J. R. (2020). Minimal dispersion approximately balancing weights: asymptotic properties and practical considerations. Biometrika, 107(1), 93-105.

Zhao, Q. \& Percival, D. (2017). Entropy balancing is doubly robust. J Causal Inference, 5(1). 